\title{
ARTICLE
}

\section{Tuning of human MAIT cell activation by commensal bacteria species and MR1-dependent T-cell presentation}

\author{
Cihan Tastan ${ }^{1,2}$, Ece Karhan ${ }^{1}$, Wei Zhou ${ }^{1}$, Elizabeth Fleming ${ }^{1}$, Anita Y. Voigt ${ }^{1}$, Xudong Yao ${ }^{3}$, Lei Wang ${ }^{3}$, Meghan Horne ${ }^{1}$, Lindsey Placek ${ }^{1}$,
} Lina Kozhaya ${ }^{1}$, Julia $\mathrm{Oh}^{1}$ and Derya Unutmaz ${ }^{1}$

\begin{abstract}
Human mucosal-associated invariant T (MAIT) cell receptors (TCRs) recognize bacterial riboflavin pathway metabolites through the MHC class 1-related molecule MR1. However, it is unclear whether MAIT cells discriminate between many species of the human microbiota. To address this, we developed an in vitro functional assay through human T cells engineered for MAIT-TCRs (eMAITTCRs) stimulated by MR1-expressing antigen-presenting cells (APCs). We then screened 47 microbiota-associated bacterial species from different phyla for their eMAIT-TCR stimulatory capacities. Only bacterial species that encoded the riboflavin pathway were stimulatory for MAIT-TCRs. Most species that were high stimulators belonged to Bacteroidetes and Proteobacteria phyla, whereas low/non-stimulator species were primarily Actinobacteria or Firmicutes. Activation of MAIT cells by high-vs low-stimulating bacteria also correlated with the level of riboflavin they secreted or after bacterial infection of macrophages. Remarkably, we found that human T-cell subsets can also present riboflavin metabolites to MAIT cells in a MR1-restricted fashion. This T-T cell-mediated signaling also induced IFNY, TNF and granzyme B from MAIT cells, albeit at lower level than professional APC. These findings suggest that MAIT cells can discriminate and categorize complex human microbiota through computation of TCR signals depending on antigen load and presenting cells, and fine-tune their functional responses.
\end{abstract}

Mucosal Immunology (2018) 11:1591-1605; https://doi.org/10.1038/s41385-018-0072-x

\section{INTRODUCTION}

Mucosal-associated invariant T (MAIT) cells are an innate-like T-cell subset abundant in human blood and mucosal tissues like the liver and intestine. ${ }^{1-4}$ MAIT cells are phenotypically defined by the expression of a semi-invariant $T$ cell receptor (TCR) (Va7.2 in humans) and the expression of CD161. ${ }^{1,2}$ MAIT cells can be activated by cells that are infected with different bacterial species and yeast. $^{3-7}$ Analyses of germ-free mice reconstituted with different bacterial species suggest that commensal flora may be necessary for both the expansion of MAIT cells in the periphery and the acquisition of a memory phenotype. ${ }^{2,4,5}$

It is now well established that in both mice and humans, MAITTCR is stimulated through the major histocompatibility complex (MHC) class I-like molecule MR1 bound to metabolites from the bacterial riboflavin pathway. ${ }^{8-10} \mathrm{~A}$ wide range of bacterial species contain this riboflavin pathway, several of which, such as Mycobacterium tuberculosis, Escherichia coli and Staphylococcus (S.) aureus, have been shown to stimulate MAIT cells. ${ }^{3,5,8}$ In contrast, bacteria that lack the genes for this riboflavin pathway, such as Enterococcus faecalis, do not stimulate MAIT cells. ${ }^{5,11}$ The specific and MR1-restricted recognition of riboflavin metabolites by MAIT cells have been shown in MAIT-TCR transgenic mice and engineered human Jurkat cell lines with invariant $\mathrm{Va}-\mathrm{Ja}$ and variable $V \beta$ segments. $2,5,8,11$

Although the role of MAIT cells in immune response is yet to be fully elucidated, they appear to play a protective role in some experimental bacterial infection models. ${ }^{6,12-14}$ For example, infection of MR1-knockout mice, where MAIT cells are absent, with Klebsiella pneumoniae resulted in a high mortality rate. ${ }^{15}$ In contrast, Va19 MAIT-TCR transgenic mice that were infected with either E. coli or M. abscessus had lower bacterial loads relative to controls. ${ }^{5,16}$ Further, in in vitro studies MAIT cells were shown to have an efficient cytotoxic effector function for bacterially infected cells. ${ }^{12,13}$ In humans, MAIT cells increase in the lungs of patients infected with the pulmonary pathogen $M$. tuberculosis (TB) and accumulation was observed in several other bacteria-infected tissues. ${ }^{3,17,18}$ In contrast to tissues, MAIT cell frequency in blood is generally reduced in humans with different bacterial infections. ${ }^{19-22}$ Collectively, these findings suggest that the infections may induce MAIT cell activation and possibly recruitment to the inflamed tissues to help for clearance of bacterial pathogens.

Several observations suggest that MAIT cells may also play a role in regulating responses to microbiota in human gut or skin and during chronic inflammatory diseases. For example, MAIT cell frequency is frequently reduced in blood of patients with inflammatory diseases such as Crohn's disease, multiple sclerosis and rheumatoid arthritis, but increased in inflamed tissue relative to healthy controls suggesting active migration to site of inflammation. ${ }^{23-25}$ The composition of the local microbiota, which could impact activation and recruitment of MAIT cells, also changes in the inflamed tissues of Crohn's disease and psoriasis. ${ }^{26-30}$ MAIT cells were also found to be reduced in the blood of HIV-infected patients in both adults ${ }^{31-35}$ and children, which display chronic inflammation partly due to perturbations in

\footnotetext{
'Jackson Laboratory for Genomic Medicine, Farmington, CT 06032, USA; ${ }^{2}$ Department of Microbiology, NYU School of Medicine, New York, NY 10016 , USA and ${ }^{3}$ Department of Chemistry, University of Connecticut, Storrs, CT 06269, USA

Correspondence: Derya Unutmaz (Derya@mac.com, JuliaOh@me.com)
}

Received: 21 August 2017 Revised: 16 July 2018 Accepted: 23 July 2018

Published online: 16 August 2018 
a

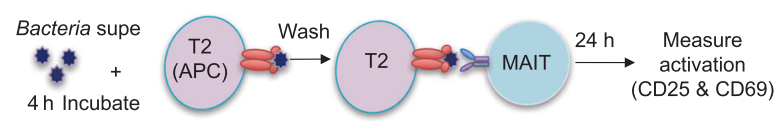

b

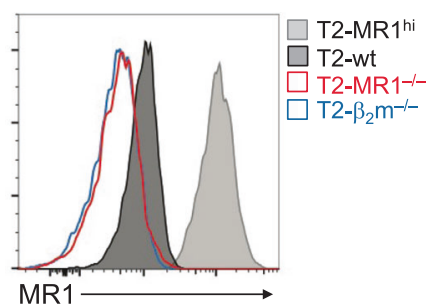

E. coli supe: $1: 10$
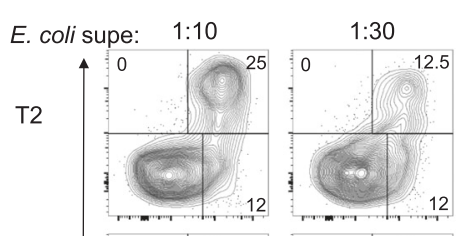

$: 30$

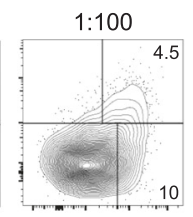

C

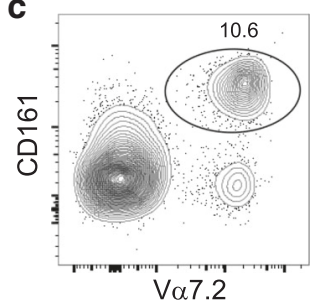

MR1 $1^{\text {hi }}$
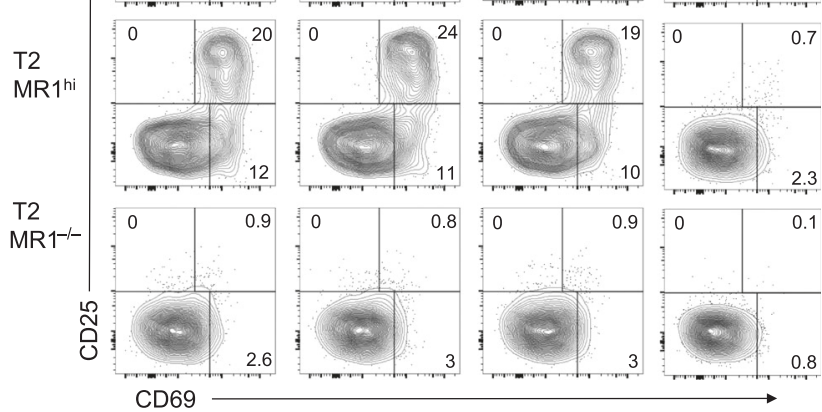

Fig. 1 Stimulation of MAIT cells through MR1-expressing cells and bacterial supernatant. a In this experimental approach, T2 cells were pre-incubated with E. coli supernatant or TSB (mock) for $4 \mathrm{~h}$. The cells were then washed twice and cultured with human adult PBMCs including MAIT cells for $16-24 \mathrm{~h}$. Activation of $\mathrm{CD} 8^{+}$MAIT cells was determined by staining with antibodies against stimulation molecules CD25 and CD69. b FACS plot of MR1 surface expression on MR1-overexpressing T2 (T2-MR1 ${ }^{\text {hi }}$, light gray filled), wild-type T2 (T2wt, dark gray filled), MR1-knockout T2 (T2-MR1 ${ }^{-1-}$, empty red line) and $\beta_{2} \mathrm{~m}$-knockout T2 (T2- $\beta 2 \mathrm{~m}^{-1-}$, empty blue line). c MAIT cell identification after gating on $\mathrm{CD}^{+}{ }^{+} \mathrm{CD} 8^{+} \mathrm{V} \alpha 7.2^{+} \mathrm{CD} 161^{+}$cell subset. d CD25 and CD69 expression on CD8 ${ }^{+}$MAIT cells in PBMCs cultured with the engineered $\mathrm{T} 2$ cell lines that were pre-incubated with either different dilutions of $E$. coli supernatant (1:10, 1:30 and 1:100) or TSB medium (1:10 dilution). Data are representative of at least three independent experiments with PBMCs from different healthy adult donors

the gut mucosal immunity and microbiota. ${ }^{37-41}$ However, it is not yet known whether MAIT cells respond to commensal human microbiota residing in mucosal tissues or skin, as to date, only selective and mostly pathogenic bacterial species are known to specifically stimulate MAIT cells. ${ }^{3,5,7,8,15}$

As such, a major knowledge gap remains in our understanding of how MAIT cells discriminate and respond to the substantial variation in the commensal human microbiota versus pathogenic bacterial species. To address this question, we developed a highly sensitive functional in vitro assay, by expression of engineered MAIT-TCRs (eMAIT-TCR) in human conventional $\mathrm{CD}^{+}{ }^{+} \mathrm{T}$ cells, to screen bacterial metabolites that may stimulate MAIT cells. We found that bacterial species from the Bacteroidetes and Proteobacteria phyla were significantly high eMAIT-TCR stimulators, whereas of Firmicutes phylum was low/non-stimulators, despite having riboflavin biosynthetic pathway. We also discovered that primary human T-cell subsets have the capacity to activate MAIT cells through bacterial metabolites and induce cytokine secretion, but at relatively lower strength of TCR signals, which correlate with their low MR1 expression. Our study suggests that MAIT cells can sense the composition of microbiota species by tuning their TCR signals, in a manner dependent on metabolite concentrations from different commensal bacterial species and expression level of MR1 on presenting cells.

\section{RESULTS}

Development of a functional assay for screening MAIT-TCR stimulation by bacteria

MAIT cell activation is mostly restricted to metabolites from bacterial riboflavin pathway, expressed by many bacterial species (both pathogenic and commensal). Thus, it remains unclear whether MAIT cells can discriminate between the composition of thousands of different commensal bacterial species in the human microbiota. However, screening hundreds or thousands of bacteria for their capacity to stimulate human MAIT cells is challenging due to their relatively small and highly variable frequency in human blood. To overcome this, we aimed to develop a robust functional assay that was highly sensitive, highthroughput, and could recapitulate activation of primary MAIT cells by diverse commensals or pathogenic bacterial species found in the human microbiota (Fig. 1a). Accordingly, we utilized a human Epstein-Barr virus-transformed and TAP (transporter associated with antigen processing)-deficient B-cell line called T2 cells ${ }^{42}$ as antigen-presenting cells (APCs), which express MR1 on cell surface (Fig. 1b). To determine the role of MR1 in this assay, we either further overexpressed MR1 on T2 cells or, using CRISPR (Clustered Regularly Interspaced Short Palindromic Repeats)/Cas9 (CRISPR associated protein 9) gene editing, deleted MR1 or B2M $\left(\beta_{2}\right.$-microglobulin $\left(\beta_{2} \mathrm{~m}\right)$ ) gene (Fig. 1b), as cell surface presentation of MR1 molecules requires expression of $\beta_{2} \mathrm{~m}$. Wild-type and $M R 1^{\text {hi }}$ and $\mathrm{MR}^{-}$T2 cells were pre-incubated with $E$. coli supernatant or tryptic soy broth (TSB) medium used for culturing E. coli, as control, washed and co-cultured with peripheral blood mononuclear cells (PBMCs) for $24 \mathrm{~h}$. Subsequently, activation of Va7.2 ${ }^{+} \mathrm{CD} 161^{+} \mathrm{CD} 8^{+}$MAIT cells in PBMCs (Fig. 1c) was assessed by upregulation of CD25 and CD69 surface molecules (Fig. 1d). Both wild-type T2 (T2-wt), which expresses MR1, and MR1overexpressing $\mathrm{T} 2$ cells (T2-MR1 ${ }^{\text {hi }}$ ) upregulated CD25 and CD69 expression specifically on CD8 ${ }^{+}$MAIT cells in PBMCs, although T2$M R 1^{\text {hi }}$ cells were more efficient, especially as $E$. coli supernatant was further diluted (Fig. 1d). In contrast, MAIT cells were not stimulated with either MR1-knockout $\mathrm{T} 2$ cells $\left(\mathrm{T} 2-\mathrm{MR} 1^{-1-}\right.$ ) preincubated with $E$. coli supernatant (Fig. 1d, bottom panel) or MR1expressing T2 cell lines with TSB controls (Fig. 1d), showing that specificity of activation is dependent on $E$. coli secreted metabolites and MR1 expression. We proceeded with subsequent experiments using $\mathrm{T} 2-\mathrm{MR}{ }^{\mathrm{hi}}$ cells as they were the most sensitive, likely due to increased capacity of MR1 to capture lower levels of metabolites in the bacterial supernatants.

Using PBMCs to screen hundreds or even thousands of bacterial species for specific MAIT-stimulatory activity is highly challenging, due to large cell number requirements and donor-to-donor variation in MAIT cell frequency and responses. ${ }^{1,2,4}$ To overcome this, we engineered a MAIT cell-specific TCR (eMAIT-TCR) with mouse TCR $\beta$ constant domain (mTCR $)$, which avoids endogenous TCR mispairing, as previously described. ${ }^{43,44}$ Accordingly, TCRinvariant Va7.2 sequence with Ja33 gene segment was paired with TCR-V $\beta 2$ domain conjugated with the J $\beta 2.1$ (TRBJ2-1) segment (Fig. 2a) for its preferential usage with the dominant MAIT-TCR-V $\beta 2 .{ }^{45-47}$ We then ectopically expressed this eMAIT-TCR on human conventional primary $\mathrm{CD}^{+} \mathrm{T}$ cells or a Jurkat cell line for comparison. ${ }^{8,11}$ The synthesized eMAIT-TCR Va-V $\beta$ gene construct was subcloned into a lentiviral vector, which contained green fluorescent protein (GFP) as a marker, and used to transduce the Jurkat cell line or activated purified human $\mathrm{CD}^{+}$ 
a

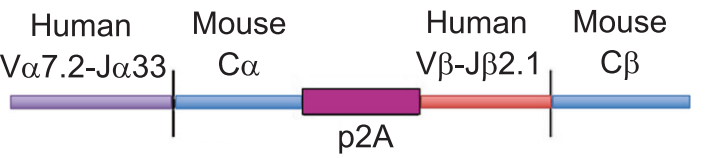

b Jurkat cells

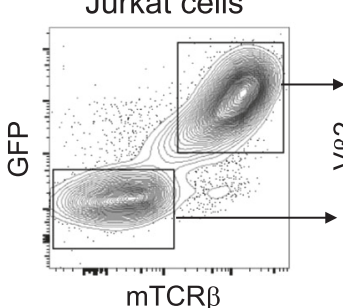

$\mathrm{mTCR} \beta$

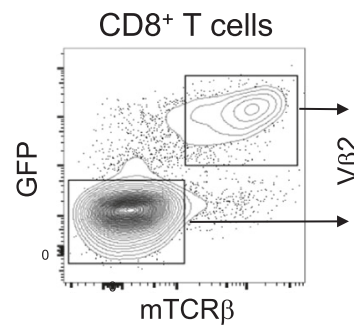

GFP+mTCR $\beta+$
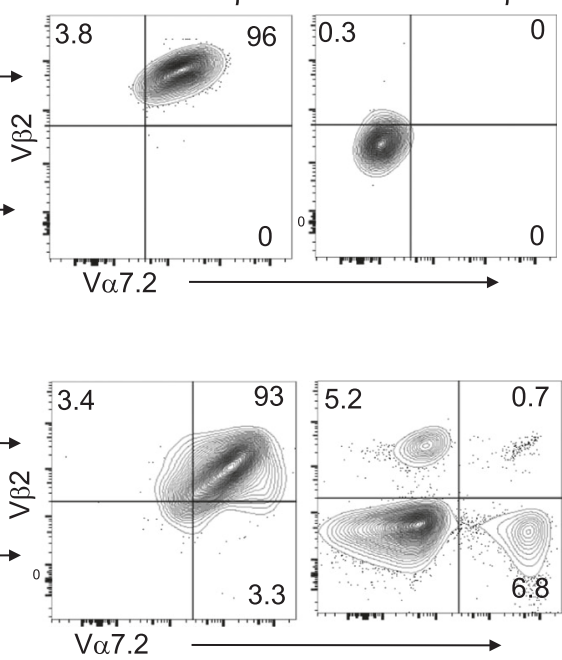

C
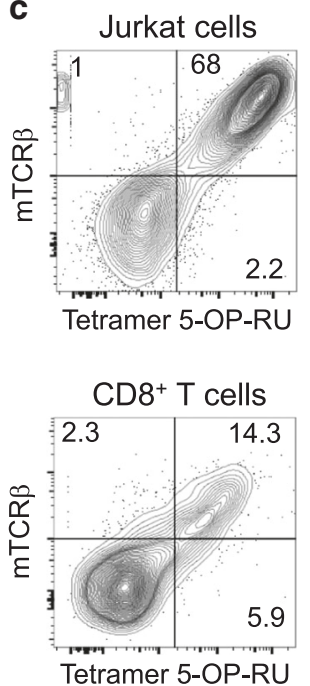

Fig. 2 Development of MR1-restricted eMAIT-TCR-expressing cells. a Construction of eMAIT-TCRs. Human TCR V $\alpha 7.2-J \alpha 33$ and V $\beta$-J $\beta 2.1$ gene segments were combined with mouse TCR constant $\alpha$ and $\beta$ segments, respectively, and linked by a picornavirus-like 2A (p2A) self-cleaving peptide sequence. b Jurkat and primary human conventional CD8 ${ }^{+}$T cells that express either eMAIT-TCR-V $\beta 2\left(G F P^{+}\right.$mTCR $\left.\beta^{+}\right)$or not $\left(\mathrm{GFP}^{-} \mathrm{mTCR} \beta^{-}\right)$, determined by staining with mTCR $\beta$-, V $\alpha 7.2$ - and V $\beta 2$-specific antibodies. c MR1 antigen complex specificity of the eMAIT$\mathrm{TCR}^{+}$cells determined by staining with 5-OP-RU pre-loaded APC-conjugated MR1 tetramer and anti-mTCR $\beta$

T cells as previously described. ${ }^{44}$ The Jurkat cell line or the expanded $\mathrm{CD}^{+} \mathrm{T}$ cells transduced with eMAIT-TCR-V $\beta 2$ encoding vector co-expressed a GFP marker and, as mentioned above, the mTCR $\beta$ which can be stained with a specific antibody (Fig. 2b). Cells gated on $\mathrm{mTCR}^{+} \mathrm{GFP}^{+}$subset expressed both Va7.2 and V 32 parts of the eMAIT-TCR in both Jurkat or $C D 8^{+}$T cells (Fig. 2b) and only $\mathrm{mTCR}^{+}$cells were stained with MR1-5-OP-RU tetramer (Fig. 2c), which specifically binds to MAIT-TCRs as described, ${ }^{11}$ and thus confirming this as MAIT-TCR.

Upon stimulation of eMAIT-TCR-V $\beta 2^{+}$cells with T2-MR ${ }^{\text {hi }}$ cells pre-incubated with $E$. coli supernatant, Jurkat cells only induced CD69, whereas primary CD8 ${ }^{+} \mathrm{T}$ cells expressing the eMAIT-TCR greatly upregulated both CD25 and CD69 expression (Fig. 3a). The eMAIT-TCR-expressing $T$ cells were also activated in a dosedependent manner by MAIT cell stimulating riboflavin metabolite (5-amino-4-D-ribitylaminouracil dihydrochloride (5-ARU)) (Fig. S1). T2 cells lacking MR1 expression (either through $M R 1$ or $\beta 2 M$, which is required for MR1 expression expression, ${ }^{48,49}$ gene-targeted CRISPR/Cas9-mediated deletion) did not activate eMAIT-TCRexpressing cells either by E. coli supernatant or 5-ARU (Fig. 3b). Together, these findings conclusively show that the activation of eMAIT-TCR ${ }^{+}$cells is MR1 dependent.

To demonstrate that eMAIT-TCR stimulation is also dependent on metabolites produced from riboflavin biosynthesis pathway of E. coli, we repressed the promoter element of ribA gene of the riboflavin pathway by CRISPRi targeting in $E$. coli since ribA is required for production of MAIT-TCR stimulatory metabolites. ${ }^{50,51}$ Repression of ribA mRNA transcription was confirmed by quantitative polymerase chain reaction ( $q P C R$ ) and resulted in a reduced capacity of $E$. coli to stimulate eMAIT-TCR-expressing T cells (Fig. 3c). Further, E. coli growth time appeared to have little impact on MAIT-stimulatory metabolite production since even $1 \mathrm{~h}$ growth culture was sufficient for optimal stimulation of eMAIT$\mathrm{TCR}^{+}$T cells (Fig. S2).
As functional outputs for TCR-mediated activation, we also measured cytokine secretion and cytotoxicity from primary $\mathrm{CD}^{+}$ $\mathrm{T}$ and Jurkat cell lines. Stimulation of eMAIT-TCR ${ }^{+} \mathrm{CD}^{+}$primary $T$ cells with $E$. coli supernatant induced high level of interferon- $\gamma$ (IFN $\gamma$ ) secretion (Fig. 3d). The primary T cells expressing eMAIT-TCR also displayed high cytotoxicity against presenting $\mathrm{T} 2-\mathrm{MR} 1^{\text {hi }}$ cells pre-cultured with either E. coli supernatant (Fig. 3e, f) or with 5ARU metabolite (Fig. 3f). In contrast, these effector functions were not observed in the stimulated eMAIT-TCR-expressing Jurkat cells (Fig. 3d, f). Thus, these findings further validate that the primary $\mathrm{T}$ cells are both more sensitive for activation and display physiologically relevant effector functions upon eMAIT-TCR stimulation.

Screening of MAIT-TCR stimulation with diverse bacterial species After establishing a proof of principle with $\mathrm{Va} 7.2^{+} \mathrm{V} \beta 2^{+}$eMAIT-TCR to expand the breadth of MAIT-TCR representation, we engineered additional TCRs that contained the constant Va7.2-Ja33 and J $\beta 2.1$ but was paired with 20 different $V \beta$ segments. These synthetic TCRs were individually expressed on $\mathrm{CD} 8^{+} \mathrm{T}$ cells and Jurkat cells, which were confirmed using V $\beta$-specific antibodies (Fig. S3). The TCR-engineered CD8 ${ }^{+}$primary T cells and Jurkat cells were then stained with MR1 tetramers pre-loaded either with 5OP-RU or 6-FP as a negative control. Only 6 of the $\mathrm{V} \beta$ segments (V $\beta$ 2, 12, 13.2, 13.5, 13.6, 20) bound MR1-5-OP-RU, albeit at differing levels (Fig. $4 \mathrm{a}, \mathrm{b}$ ), possibly due to changes in overall TCR affinities. We then tested all TCRs for their capacity to signal in $T$ cells using functional assay described above. We found that only 7 of the engineered TCRs (eTCRs; all those stained with MR1-5-OP$\mathrm{RU}$ and $\mathrm{V} \beta$ 13.1) were also stimulated by $E$. coli supernatant (Fig. 4c), and that staining intensity of these with MR1-5-OP-RU correlated with the proportion of cells activated (compare Fig. $4 \mathrm{~b}$ with Fig. 4c). In fact, the differences between strength of activation of these 7 eTCRs were much more evident when they were 


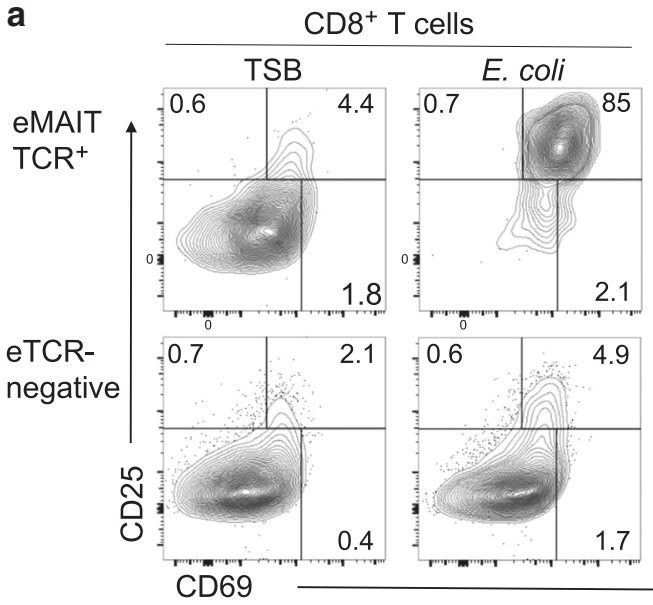

b
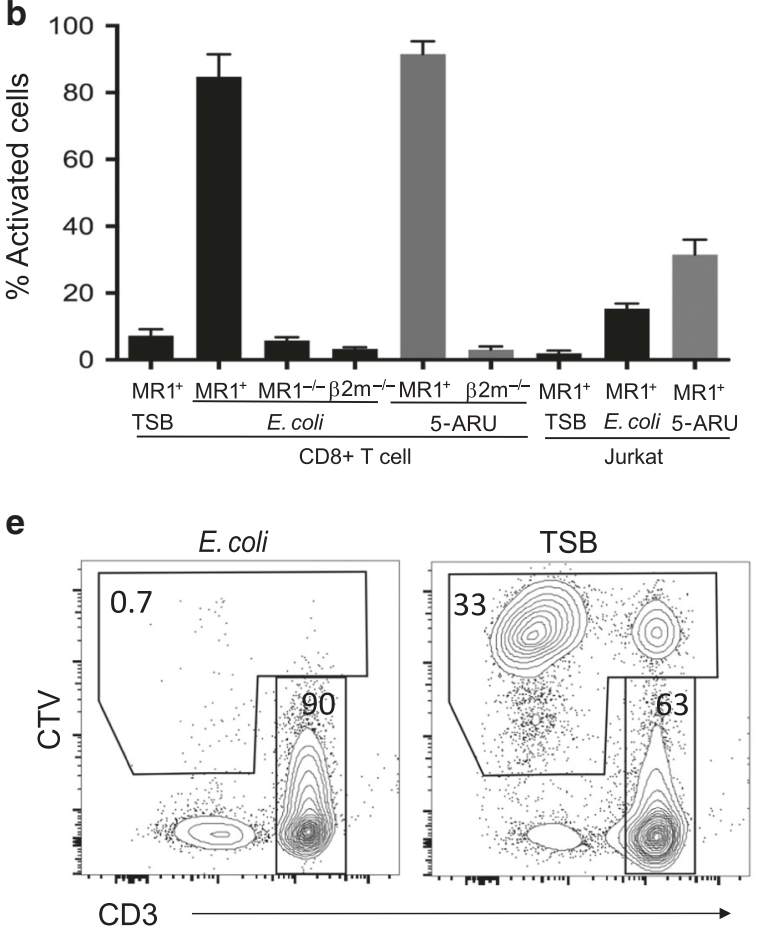

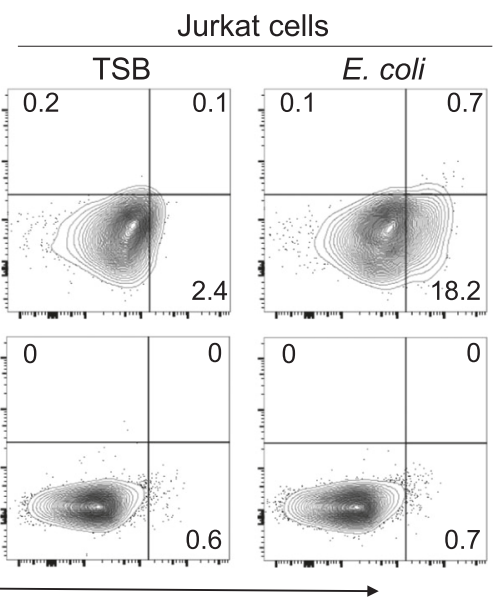

C

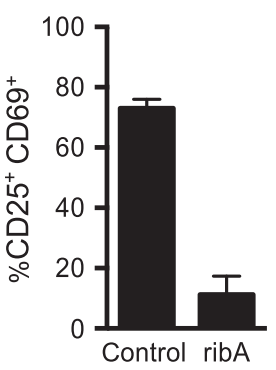

d
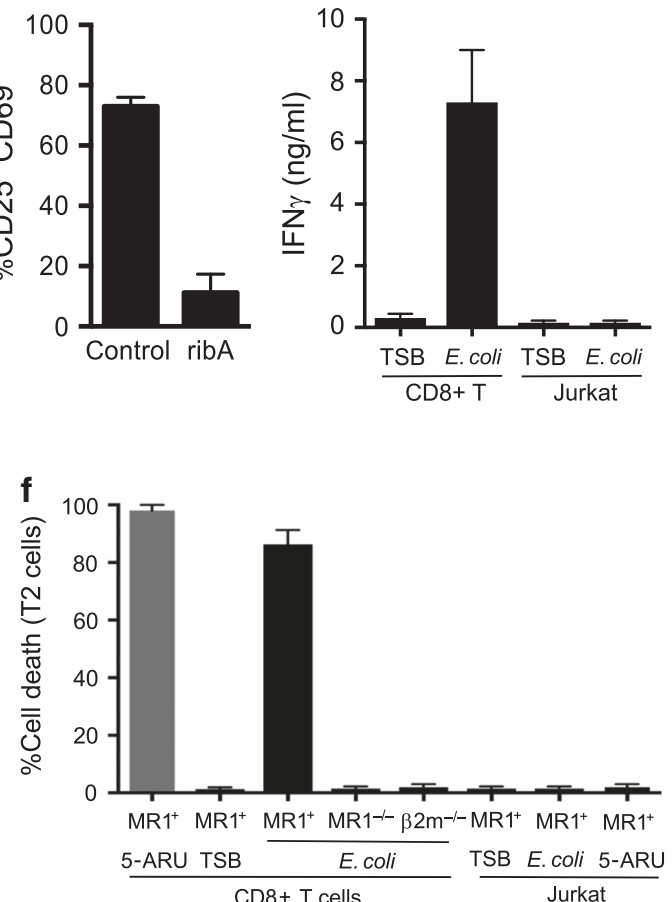

Fig. 3 Effector functions of MR1-restricted eMAIT-TCR ${ }^{+}$T-cell activation. a CD25 and CD69 upregulation of stimulated eMAIT-TCR-V $\beta 2^{+}$CD8 ${ }^{+}$ $\mathrm{T}$ or Jurkat cells upon culturing with T2-MR1 ${ }^{\text {hi }}$ cells and $E$. coli supernatant compared to eMAIT-TCR-negative cells in the same culture or eMAIT-TCR ${ }^{+}$cells in TSB control condition. b Quantification of the stimulated eMAIT-TCR-V $\beta 2^{+}$CD8 ${ }^{+}$T cells $\left(\% C D 69^{+}\right.$CD25 $5^{+}$or Jurkat cells $(\%$ $\mathrm{CD} 9^{+}$) that were cultured with the T2 cells (MR1 ${ }^{\mathrm{hi}}, \mathrm{MR}^{-/-}$or $\beta_{2} \mathrm{~m}^{-/-}$) and E. coli supernatant, or 5-ARU compound or TSB. c Stimulation of eMAIT-TCR ${ }^{+}$T cells cultured with T2-MR $1^{\text {hi }}$ cells and supernatants from $E$. coli clones that were transformed with either ribA-targeting CRISPR or control non-targeting CRISPR plasmid. d Assessment of IFN $\gamma$ secretion upon stimulation of eMAIT-TCR ${ }^{+} \mathrm{T}_{\text {cells }}$ using cytokine bead array (CBA). e FACS plots of effector CD3 ${ }^{+}$eMAIT-TCR-expressing T cells and T2 cells were labeled with Cell Trace violet (CTV) fluorescent molecule. $f$ Quantification of the viable portion of the T2 cells (MR1 ${ }^{\text {hi }}, \mathrm{MR}^{-1-}$ or $\beta_{2} \mathrm{~m}^{-/-}$) after culturing with eMAIT-TCR-V $\beta 2^{+}$CD8 ${ }^{+}$T or Jurkat cells in the presence of $E$. coli supernatant or 5-ARU compound or TSB. Means and standard error range of at least three independent experiments are shown

activated with varying concentrations of 5-ARU and activation was measured as IFNY secretion (Fig. 4d), which showed remarkably similar hierarchy of V $\beta$ MAIT-TCR activation both with $E$. coli supernatant and in MR1 tetramer staining intensity (Fig. 4d, middle and right panels). Henceforth, we refer to these 7 functional eTCRs as eMAIT-TCRs, which were subsequently used in the functional bacterial screens as described below.

We next screened 47 human-associated bacterial species in the eMAIT-TCR assay for their stimulatory capacity. These were comprised primarily of commensals and belonging to different phyla present in different mucosal tissues as commensals, in addition to a few environmental strains potentially present in different human tissues (Fig. 5a, tissue localization column) based on $16 \mathrm{~S}$ ribosomal RNA and shotgun metagenomic studies. ${ }^{52,53}$ The eMAIT-TCR with V 32 was the most sensitive for detecting stimulation with supernatants from the bacterial species, with more variable activation of other engineered TCRs, assessed by CD25 and CD69 (Fig. 5a, bars under each eMAIT-TCR). This was again consistent with their binding patterns to MR1 tetramers (Fig. 4a, b). Species that lacked riboflavin pathway also completely lacked stimulatory capacity for any of the eMAIT-TCRs (Fig. 5a, gray strip). To more broadly quantify the capacity of different 
a

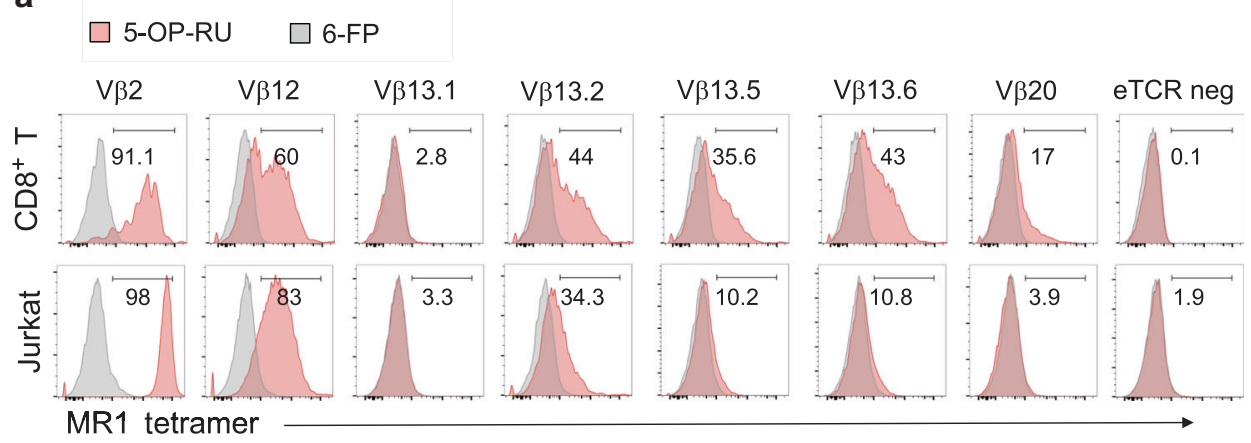

b

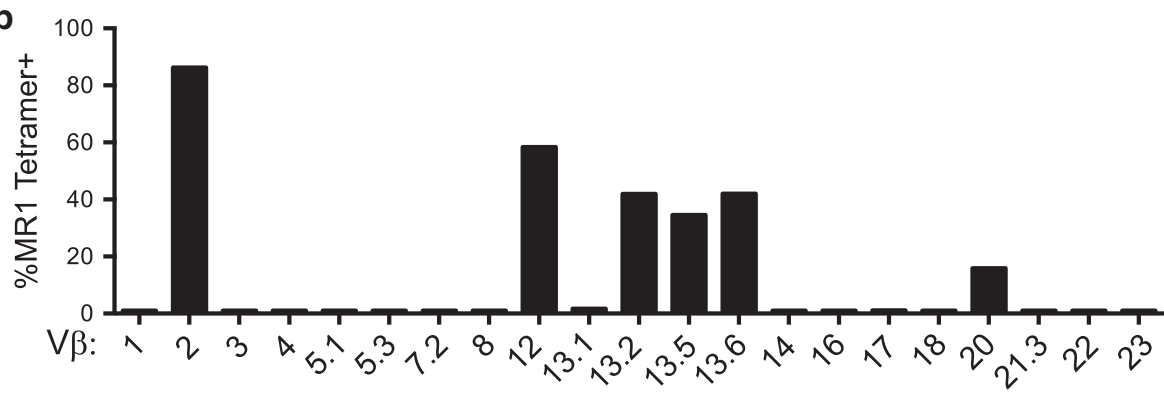

C

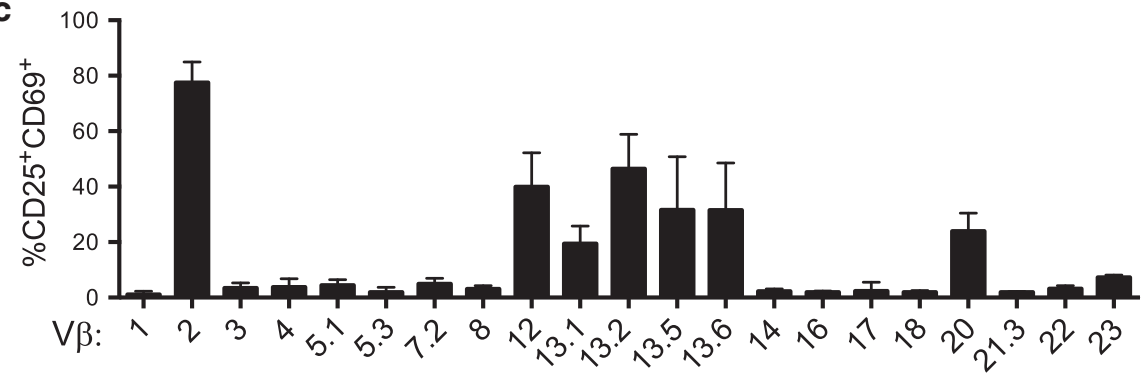

d

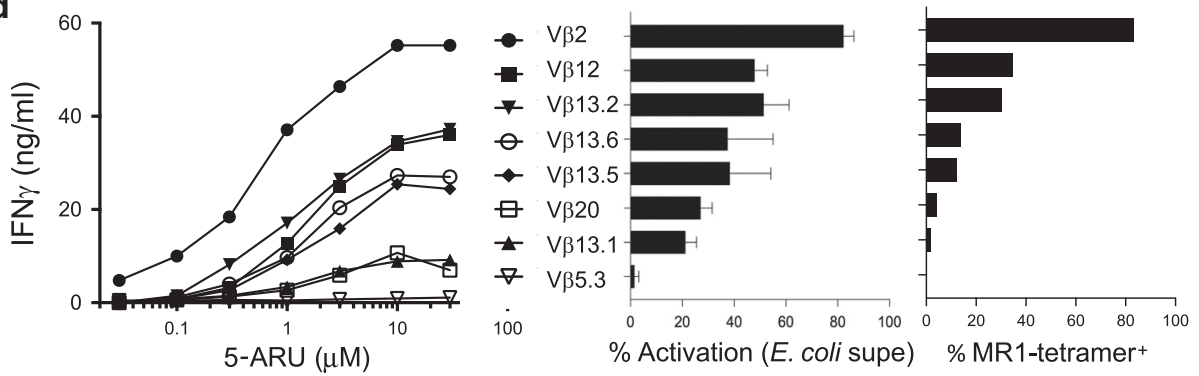

Fig. 4 MR1-restricted expression and stimulation of eMAIT-TCRs. a 5-OP-RU or 6-FP (negative control) pre-loaded MR1 tetramer staining of $\mathrm{CD} 8^{+} \mathrm{T}$ and Jurkat cells that express eTCRs with diverse $\mathrm{V} \beta$ domains after gating on $\mathrm{GFP}^{+} \mathrm{mTCR}^{+}$subset. $\mathbf{b}$ Quantified tetramer binding of $\mathrm{CD}^{+}{ }^{+}$cells expressing eTCRs with 21 different V $\beta$ segments after subtracting the basal level of binding detected with MR1-6-FP tetramer staining. c Activation of eTCR-V $\beta$-expressing T cells with T2-MR ${ }^{\text {hi }}$ and E. coli supernatant, as determined by staining for CD25 and CD69 expression on T cells. d Activation of functional eMAIT-TCR-expressing T cells with 5-ARU with T2 cells, determined by IFN $\gamma$ secretion in supernatants after 2 days of stimulation. Activation levels of each eMAIT-TCR with $E$. coli supernatant from (c) and quantified tetramer binding capacities of each eMAIT-TCR from (b) is shown on the right side of the figure corresponding to each V $\beta$ family eMAIT-TCR for comparison. Means and standard deviation of at least three independent experiments are shown

bacteria for their eMAIT-TCR stimulatory capacity, we added the percent activation by each eMAIT-TCR for each species to create a cumulative stimulation index (Fig. 5b). This analysis showed remarkable clustering of bacterial species based primarily on phyla (Fig. 5b, c) despite significant phylogenetic distance between the species themselves. Indeed, species belonging to Bacteroidetes and Proteobacteria had statistically higher MAIT-TCR stimulation capacity compared to species Firmicutes or Actinobacteria (Fig. 5c). Importantly, measuring activation, by representative bacterial supernatants from each phylum, of primary $\mathrm{CD}^{+}$MAIT cells in healthy adult PBMCs from different donors recapitulated their respective stimulatory capacity (Fig. $5 \mathrm{~d}$ ) and correlated with cumulative stimulation of eMAIT-TCR ${ }^{+} \mathrm{CD}^{+}$T cells (Fig. 5e). These findings suggest that activation of eMAIT-TCRs by diverse bacterial supernatants reflects their stimulatory capacity for primary MAIT cells.

To rule out the possibility that the eMAIT-TCR or primary MAIT cell activation could be affected by different bacterial density or concentration, given different growth kinetics for each species, we counted each species at their stationary phase as described in the 


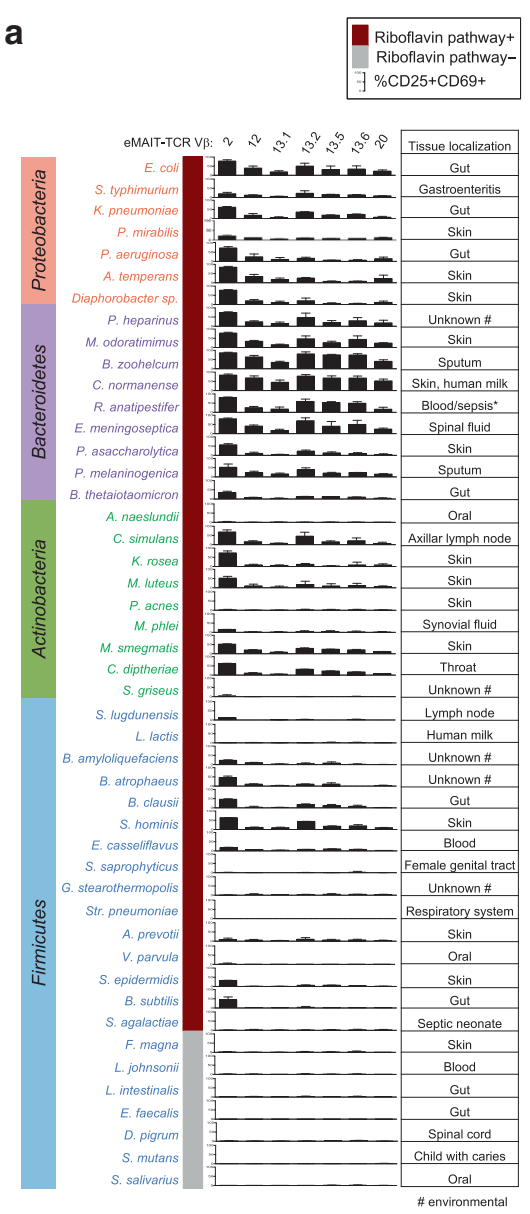

\section{a}

C
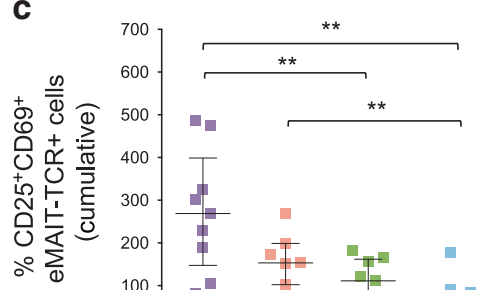

Bacteroidetes - Proteobacteria Actinobacteria
Firmicutes

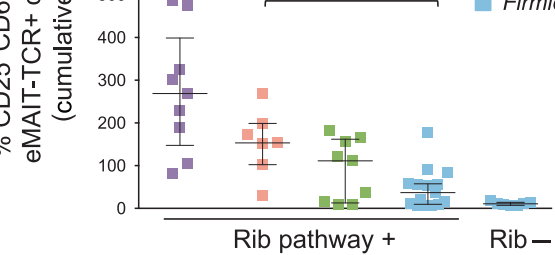

e

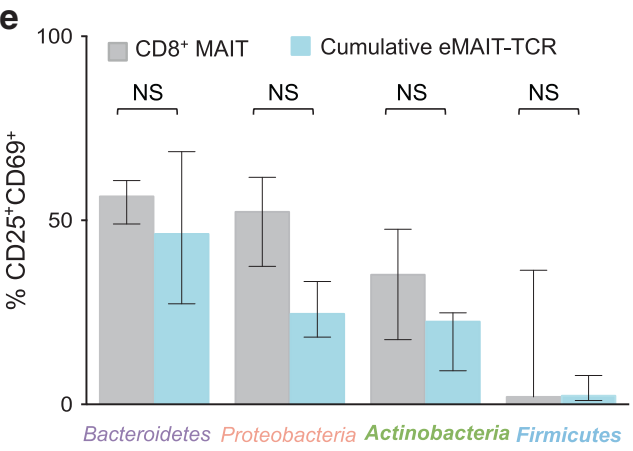

Materials and methods. While there was nearly 3-log difference in concentration among different species, there was no significant correlation between the bacterial counts and eMAIT-TCR stimulation (Fig. 5f), which is also consistent with the time course experiment using $E$. coli cultures where metabolite concentrations
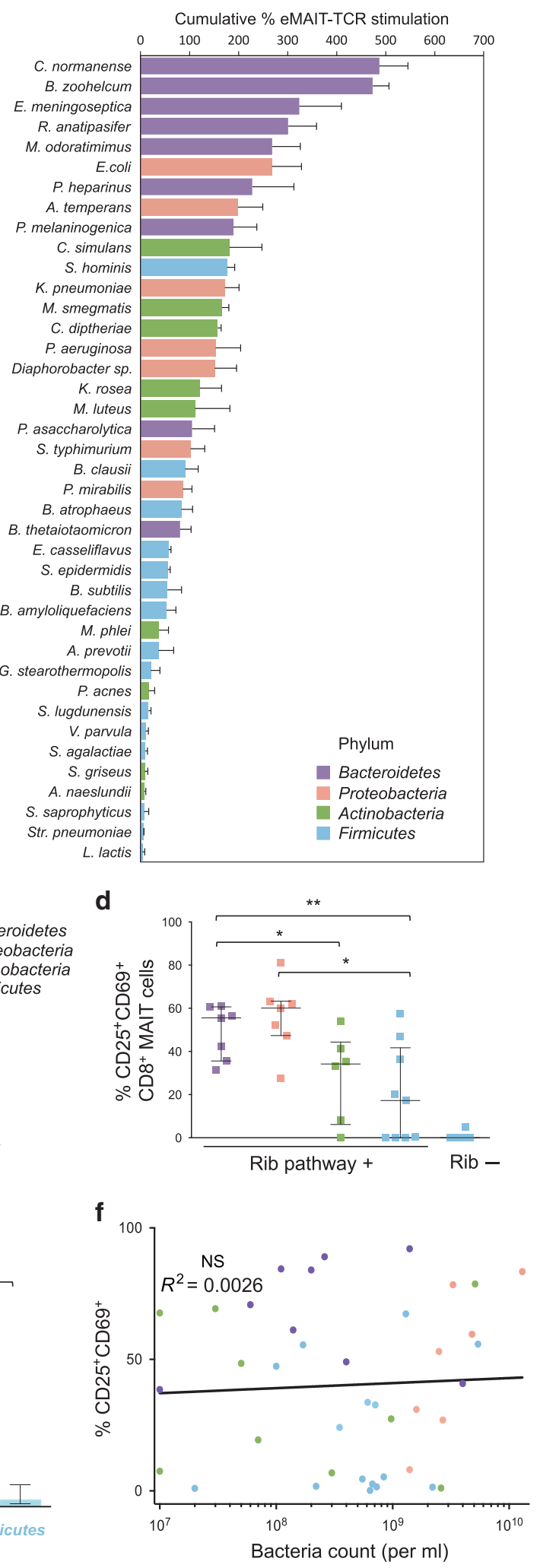

quickly reached high levels sufficient for MAIT-TCR activation before stationary phase (Fig. S2).

These findings suggested that bacteria could possess the riboflavin pathway yet not synthesize sufficient levels of riboflavin or secrete the metabolites that are required for MAIT 
Fig. 5 Screening diverse bacteria strains for their eMAIT-TCR stimulation capacity. a Bacterial species clustered and color-coded based on phylum-level classification (red strip, riboflavin pathway-positive strains; gray strip, riboflavin pathway-negative strains). The chart plot shows $\mathrm{CD}_{25}{ }^{+} \mathrm{CD} 9^{+} \mathrm{T}$-cell percent, after each bacterial species in stimulating eMAIT-TCRs $(\mathrm{V} \beta 2,12,13.1,13.2,13.5,13.6,20)$ tested. Basal level of $\mathrm{CD} 25^{+} \mathrm{CD} 69^{+}$cells was subtracted from each stimulation condition. "Tissue localization" column indicates the places of species detected in human or found in environment (\#). Means and standard error range of at least three independent experiments are shown. $\mathbf{b}$ Cumulative eMAIT-TCR stimulation index was calculated by adding total percent stimulation of eMAIT-TCRs with 7 functional V $\beta$ domains (V $\beta 2$, 12, 13.1, $13.2,13.5,13.6,20)$ from (a) (added to $700 \%$ ) for each bacterial species possessing riboflavin pathway. The species were color-coded based on phylum. Comparison of the clustered bacterial species from different phyla by stimulation capacities of either c cumulative eMAIT-TCR ${ }^{+} \mathrm{T}_{\text {cells }}$ (Bacteroidetes $n=9$, Proteobacteria $n=7$, Actinobacteria $n=9$, Firmicutes $n=15$ and Firmicutes that lacks riboflavin pathway or Rib ${ }^{-1-} n=7$ ) or $\mathbf{d}$ $\mathrm{CD}^{+}$MAIT cell activation in PBMCs by bacteria supernatants (Bacteroidetes $n=7$, Proteobacteria $n=7$, Actinobacteria $n=6$, Firmicutes $n=9$ and Firmicutes that lacks Riboflavin pathway, $\mathrm{Rib}^{-1-} n=5$ ). e Comparison between stimulation levels of primary CD8 ${ }^{+}$MAIT cells (gray filled) and cumulative eMAIT-TCR repertoire ${ }^{+}$T cells (cumulative activation normalized to $100 \%$ scale, blue filled) tested with same representative species from each phylum. Center line denotes sample median with interquartile ranges $\left(* P<0.05 ;{ }^{*} P<0.01\right.$; NS not significant using two-tailed unpaired Mann-Whitney rank sum $U$-test). f Correlation between activation of eMAIT-TCR-V $\beta 2^{+} \mathrm{T}$ cells and the bacterial numbers of the tested species having the riboflavin pathway (NS not significant, $R^{2}=0.0026$, Spearman's rank test). Same color palette representing different phyla was used in each of the above figures as shown in (b) or (c)

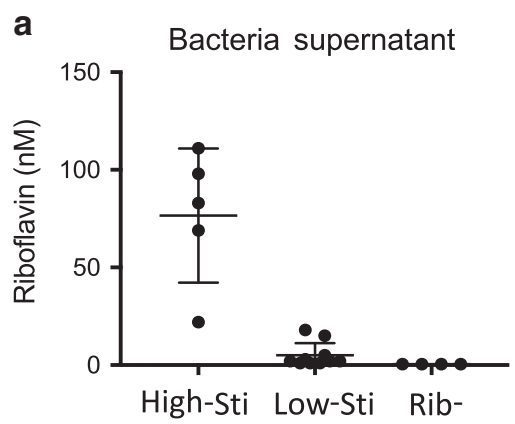

C
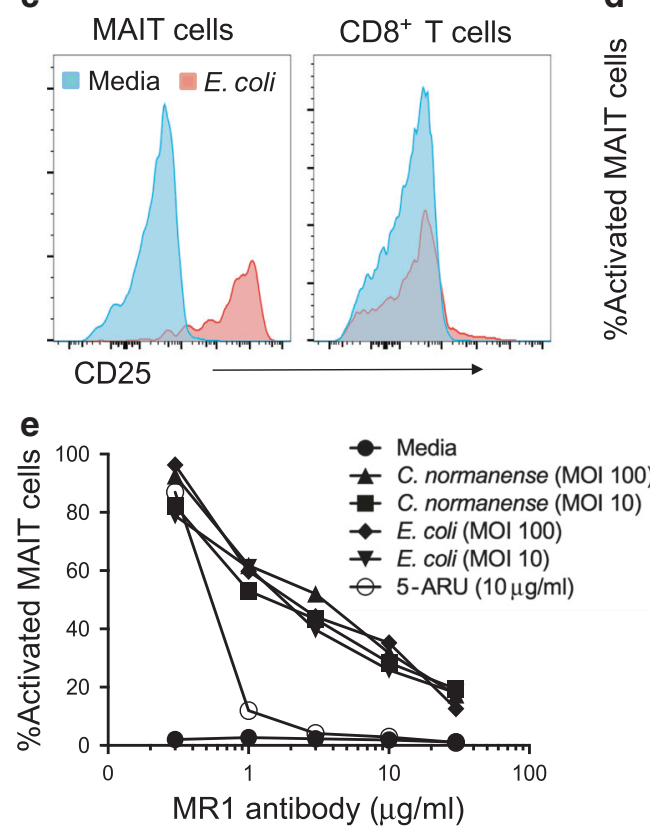
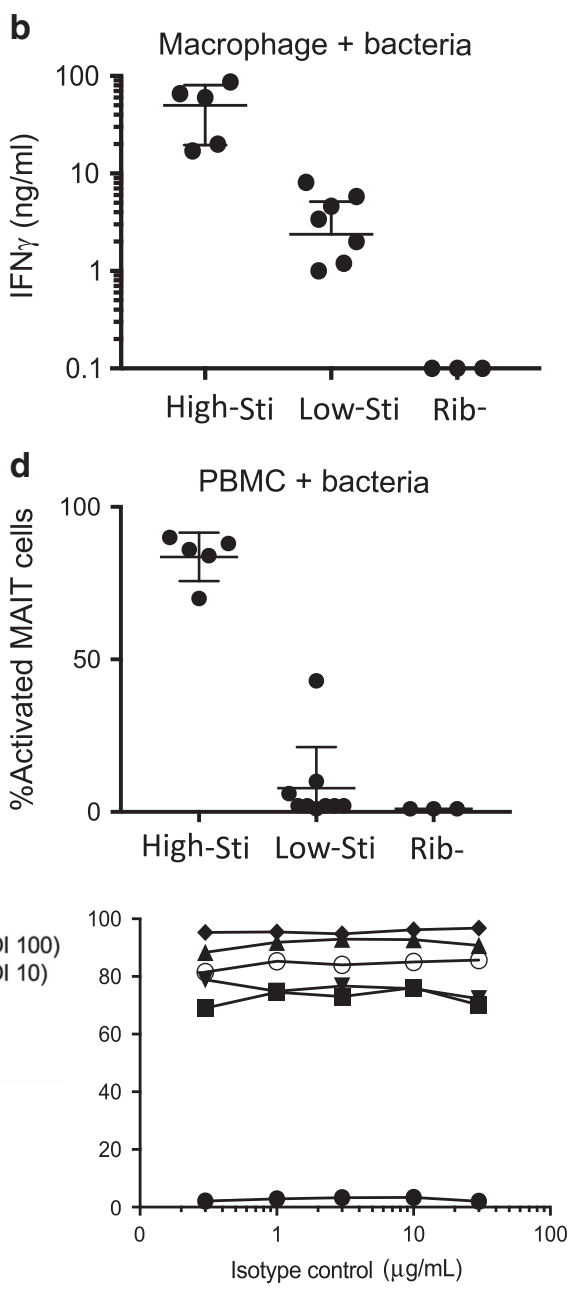

Fig. 6 Stimulation of MAIT cells through antigen-presenting cells co-cultured with bacteria. a Riboflavin levels in bacteria supernatants, grouped as high $(n=5)$ or low $(n=10)$ stimulators (cumulative activation $>200$ based or $<100$ respectively, based on Fig. $5 b)$ that contain Riboflavin pathway (Rib+), or those without (Rib-, $n=4)$. b IFN $\gamma$ secretion from eMAIT cells after 2-day stimulation with macrophages cultured in high $(n=5)$ or low $(n=7)$ stimulator or those without Rib- $(n=3)$ bacteria (at 1:100 macrophage to bacteria ratio). c Activation of primary MAIT cells (gated as CD3 ${ }^{+} \mathrm{CD}^{+} \mathrm{CD} 161^{+} \mathrm{V} \alpha 7.2^{+}$) or non-MAIT T cells $\left(\mathrm{CD} 3^{+} \mathrm{CD} 8^{+} \mathrm{CD} 161^{-} \mathrm{Va} 7.2^{-}\right.$) after culture of PBMC with $\mathrm{E}$. coli and $\mathbf{d}$ selected high $(n=5)$ or low $(n=9)$ stimulator or those without Rib- $(n=3)$ bacterial species from Fig. 5 . e Blocking of MAIT cell activation by an MR1 antibody. Two high stimulatory bacteria (E. coli and C. normanense at MOI: 100 or 10$)$ or 5-ARU (10 $\mu \mathrm{M})$ were added to PBMCs preincubated with the MR1 antibody or isotype control and activation was determined by total CD $25^{+}$CD $69^{+}$expression. Data are representative of three independent experiments with different PBMCs from healthy individuals 
a

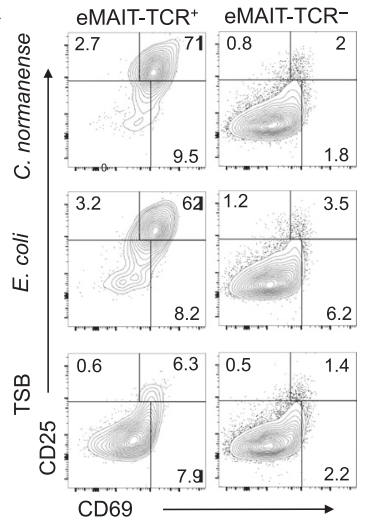

C

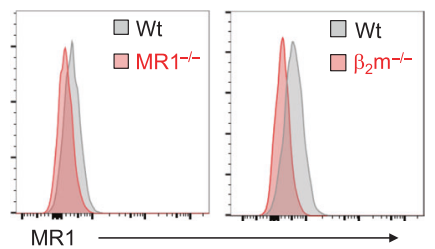

b

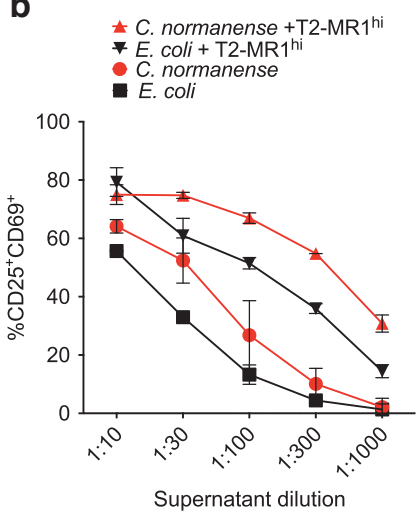

d

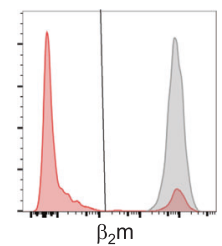

h

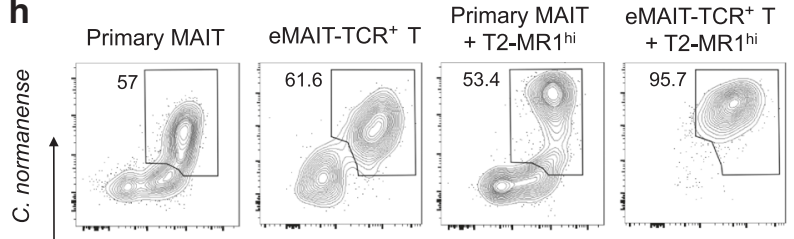

호

18
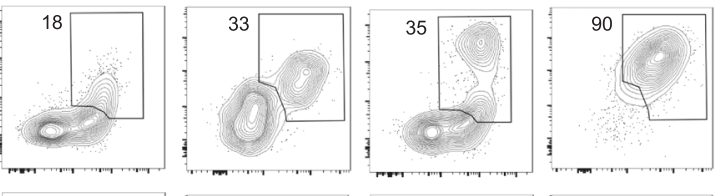

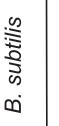
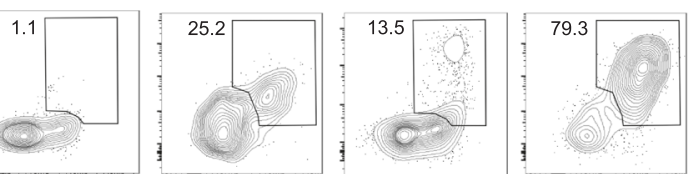

$\frac{}{\frac{\pi}{0}}$
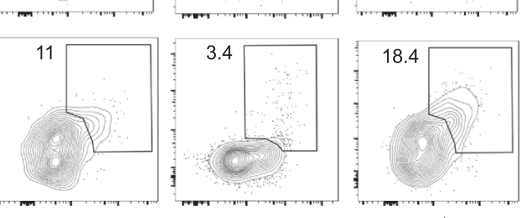

e

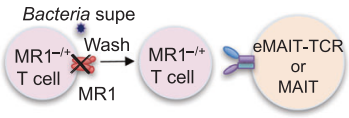

f

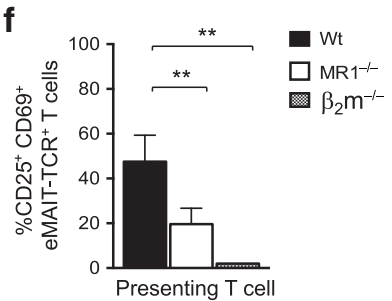

g

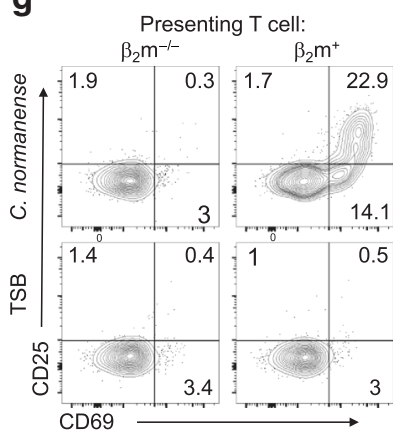

Fig. 7 Stimulation of MAIT-TCR ${ }^{+}$cells through T-cell presentation of bacteria supernatants . a FACS plots of CD25 and CD69 expression in eMAIT-TCR-V $\beta 2^{+} \mathrm{CD}^{+} \mathrm{T}$ cells incubated with either $E$. coli or $C$. normanense supernatants and TSB control in the absence of T2 cells. b Quantitative stimulation of eMAIT-TCR ${ }^{+}$T cells in the presence or the absence of T2-MR $1^{\text {hi }}$ cells with dose dependence of $C$. normanense (red

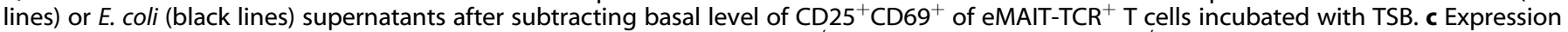
of MR1 on T cells that were either wild type (Wt, gray), MR1-deleted (MR1 ${ }^{-1-}$, red) or $\beta 2 \mathrm{~m}$-deleted $\left(\beta 2 \mathrm{~m}^{-\prime-}\right.$, red) T cells. d $\beta_{2} \mathrm{~m}$ expression in Wt or $\beta 2 \mathrm{~m}^{-1-}$ T cells. The $\beta 2 \mathrm{~m}$-negative (deleted) T-cell subsets were sorted as shown (red portion). e Experimental setup to assess stimulation of either eMAIT-TCR ${ }^{+}$T cells or MAIT cells in the purified $\mathrm{CD}^{+}{ }^{+}$T cells that were cultured with $\mathrm{CD} 4^{+}$or $\mathrm{CD} 8^{+} \mathrm{T}_{\text {cells }}\left(\mathrm{wt}, \mathrm{MR}^{-1-}\right.$ or $\beta 2 \mathrm{~m}^{-1-}$ ) preincubated with C. normanense supernatant. $\mathbf{f}$ Quantification of the activation level of eMAIT-TCR ${ }^{+}$T cells via the T-MAIT cell stimulation after subtracting basal level of $\mathrm{CD}_{2} 5^{+} \mathrm{CD}^{+} 9^{+}$in TSB $\left({ }^{*} P<0.01\right.$ using two-tailed unpaired Mann-Whitney rank sum U-test). g CD25 and CD69 expression in the primary MAIT cells within purified CD8 ${ }^{+} \mathrm{T}$ cells cultured with sorted $\beta 2 \mathrm{~m}^{-1-}$ or $\beta 2 \mathrm{~m}^{+} \mathrm{CD} 4^{+} \mathrm{T}$ cells. $\mathbf{h}$ CD25 and CD69 expression in primary MAIT cells in purified CD8 ${ }^{+}$T cells and eMAIT-TCR-V $\beta 2^{+}$T cells in the presence or absence of T2-MR $1^{\text {hi }}$ cells preincubated with C. normanense, E. coli, B. subtilis supernatants or control growth media (TSB or nutrient broth). Percent of activated T cells $\left(\mathrm{CD} 25^{+} \mathrm{CD} 69^{+}\right)$is shown in each plot

cell activation. To confirm this, we measured riboflavin secretion from a select group of high and low MAIT-stimulating bacteria that possessed the riboflavin pathway using mass spectrometry (mass spec) analysis. Those lacking the pathway were used as controls. For this experiment, the bacteria were grown overnight and then washed to remove the media, counted and resuspended in phosphate-buffered saline (PBS) at similar numbers and incubated for an additional $2 \mathrm{~h}$. The mass spec analysis of riboflavin in bacterial supernatants were then performed as described in detail in the Materials and methods section. Indeed, the high-stimulator bacterial species secreted greater amount of riboflavin compared to low-stimulator bacteria (Fig. 6a).

However, it is also possible that MR1-binding metabolites produced by some bacteria may not be as stable. To address this, we added bacteria directly on to primary human macrophages in the presence of human serum. In this assay, bacteria would be taken up by the macrophages and their metabolites then bind to MR1 in macrophages and be presented to eMAIT-TCR cells. We then measured secretion of IFN $y$ from the eMAIT-TCR cells after co-culturing with macrophages and bacteria. Remarkably, the activation of $\mathrm{T}$ cells as measured by IFN $\gamma$ secretion again was greater with high- vs low-stimulator bacteria or those that lacked the riboflavin pathway (Fig. 6b). In a similar experimental approach, we also added the bacteria directly to PBMCs to test activation of primary MAIT cells. We found a similar activation profile, as assessed by specific upregulation of CD25 (Fig. 6c), only on primary MAIT cells with high- vs low-stimulator bacteria (Fig. 6d) but not in any non-MAIT CD8 ${ }^{+}$T cells which did not show any activation in the presence of the bacteria. To further confirm that this was MR1-dependent T-cell activation, we used a blocking MR1 antibody which completely abolished the activation of 
a

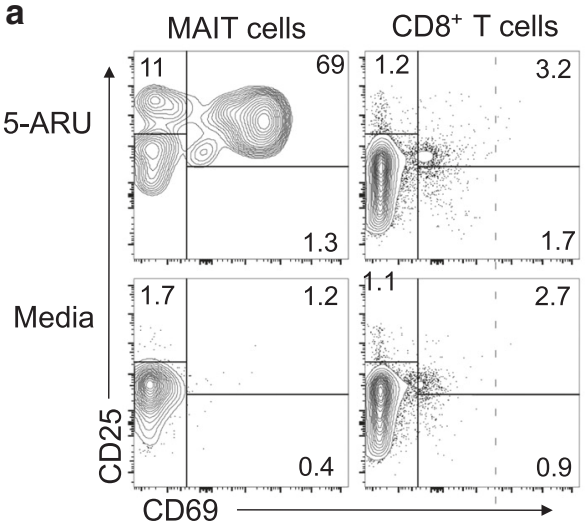

b

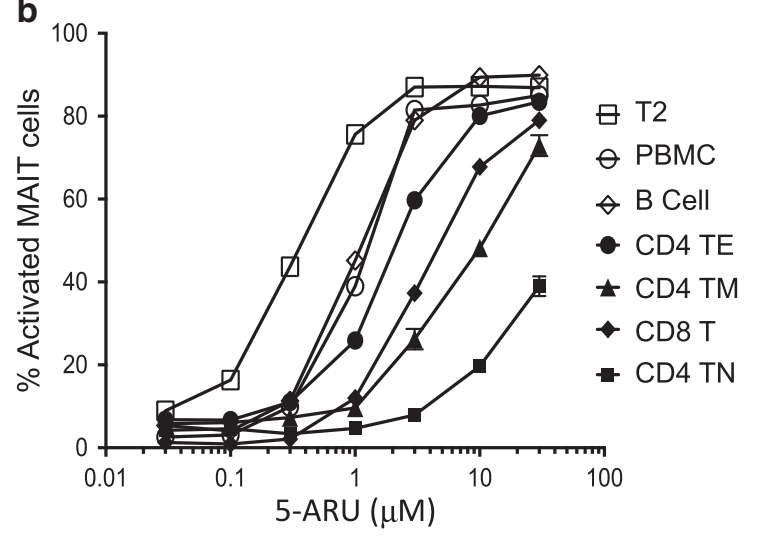

C

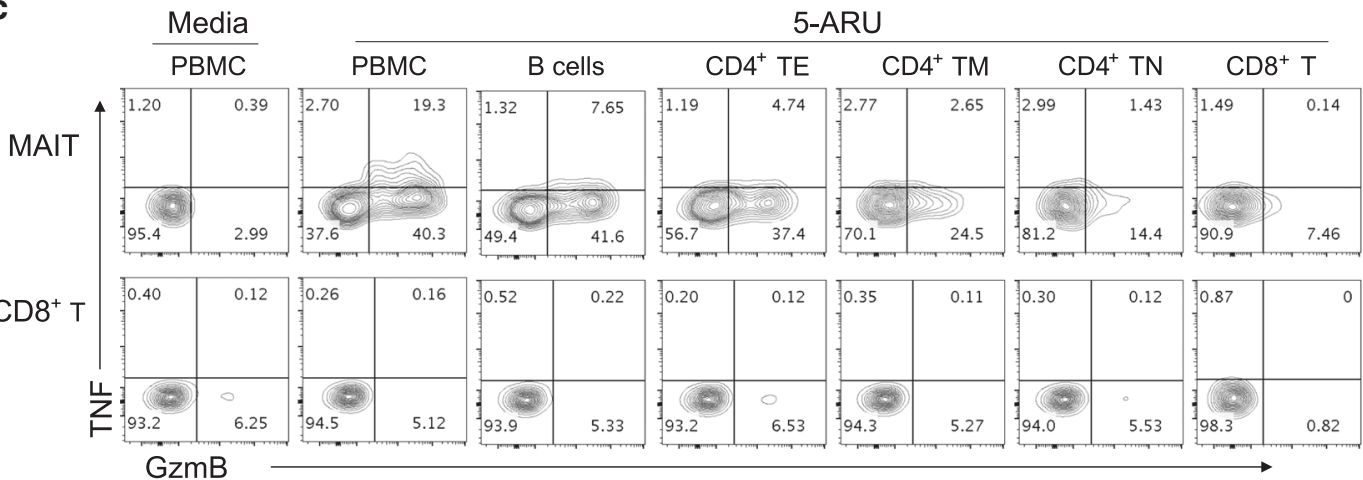

d
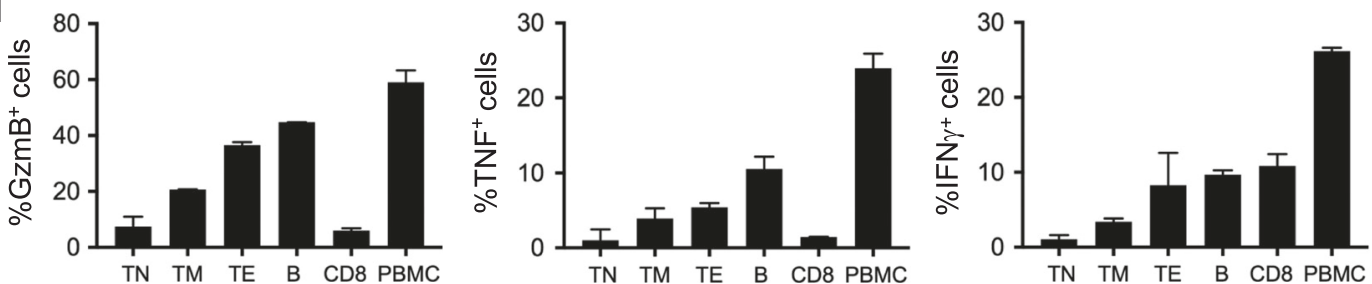

Fig. 8 Activation thresholds of MAIT cells through MR1-restricted 5-ARU compound presented by T-cell subsets. a Activation of MAIT cells in PBMCs (gated as $\mathrm{CD} 3^{+} \mathrm{CD} 8^{+} \mathrm{CD} 161^{+} \mathrm{Va7.2}{ }^{+}$) or non-MAIT cells (gated as CD3 ${ }^{+} \mathrm{CD} 8^{+} \mathrm{CD} 161^{-} \mathrm{V} \alpha 7.2^{-}$), assessed by CD25 or CD69 expression

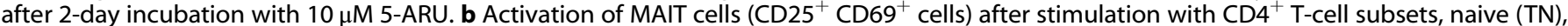
memory (TM), effector/activated (TE) and B cells pre-incubated with different concentrations of 5-ARU, washed and added to PBMCs that contained MAIT cells ( 2\%). The compound was also added directly to PBMCs (shown as PBMCs) or purified CD8 ${ }^{+} \mathrm{T}$ cells that contain most MAIT cells. c PBMCs activated as in (b) were stained intracellularly for granzyme B (GzmB), IFN $\gamma$ and TNF along with cell surface markers to define MAIT cells, and data shown are cells gated on MAIT cells $\left(\mathrm{CD} 3^{+} \mathrm{CD} 8^{+} \mathrm{CD} 161^{+} \mathrm{V} \alpha 7.2^{+}\right)$or non-MAIT cells gated as $\left(\mathrm{CD}^{+} \mathrm{CD} 8^{+} \mathrm{CD} 161^{-} \mathrm{V} \alpha 7.2^{-}\right)$. d Analysis of data from (c), as proportion of MAIT cells that express GzmB, TNF or IFN $\gamma$ (after subtracting background of non-stimulated cells) upon stimulation of different cell types. There was no difference between the background and after stimulation in non-MAIT T-cell subsets in the same PBMC (data not shown). Data are representative of three independent experiments with different PBMCs from healthy adult donors

primary MAIT cells compared to isotype control antibody (Fig. 6e). Together, these findings suggest that, at least for the bacteria tested in these experiments, the level of riboflavin production appeared to be the main driver of their capacity to stimulate MAIT cells.

Stimulation of MAIT-TCR by bacterial antigens in the absence of APCs

During the course of eMAIT-TCR stimulation assays, we observed that supernatants from high stimulatory bacteria $(E$. coli or $C$. normanense) could activate eMAIT-TCR-expressing cells alone, that is, in the absence of $\mathrm{T} 2$ cells (Fig. $7 \mathrm{a}, \mathrm{b}$ ). This was a surprising result and suggested that primary human $T$ cells may express sufficient level of MR1 for T-T cell presentation of bacterial metabolites. Although expression of MR1 in primary T cells was very low, deletion of either $M R 1$ or $\beta 2 M$ gene (as mentioned above, required for MR1 cell surface expression) using CRISPR/Cas9 gene editing in primary $T$ cells resulted in reduction in intensity of MR1 antibody staining (Fig. 7c). Furthermore, MR1 expression was increased upon incubation of the cells with 5-ARU (Figure S4), using a previously described protocol, ${ }^{54}$ suggesting that primary T cells can express MR1 on cell surface. Importantly, deletion of $\beta_{2} m$ also allowed us to negatively sort the $\beta_{2}$ m-deleted cells (Fig. $7 d$ ) to generate primary $T$ cells completely devoid of MR1 cell surface expression. Indeed, we found that $\beta_{2} \mathrm{~m}^{-/-}$sorted $\mathrm{CD} 4^{+}$or $\mathrm{CD} 8^{+} \mathrm{T}$ cell subsets, pre-incubated with the bacterial supernatants as APCs (Fig. 7e), did not stimulate eMAIT-TCR ${ }^{+} \mathrm{CD}^{+}{ }^{+}$cells (Fig. 7f) or primary MAIT cells (Fig. $7 \mathrm{~g}$ ). This demonstrates that $\mathrm{T}$ cells express sufficient level of MR1 to present bacterial metabolites to trigger MAIT-TCRs. 
We next compared three different bacterial species $(C$. normanense, E. coli and B. subtilis) with different MAIT cell stimulatory capacity in the absence or presence of T2-MR $1^{\text {hi }}$ cells. While both primary MAIT cells within CD8 + T cells or eMAIT-TCR ${ }^{+}$ $T$ cells were stimulated in the presence or absence of T2-MR $1^{\text {hi }}$ cells with $C$. normanense or $E$. coli supernatants, the activation level based on CD25 expression was higher in the presence of T2$M R 1^{\text {hi }}$ cells. Activation of primary MAIT cells alone in the presence of $B$. subtilis was much lower or undetectable (Fig. 7h). These experiments using bacteria supernatants revealed a gradient of MAIT-TCR stimulation strengths from resting $C D 8^{+} \mathrm{T}$ cells (primary MAIT) and effector eMAIT-TCR ${ }^{+}$cells to T2 cells overexpressing MR1 that was also dependent on stimulation capacity of the bacterial species.

To better assess and compare the presenter capacity of primary $T$ cells, we next repeated similar experiments using 5-ARU metabolite as the antigen. In these experiments, we further sorted $\mathrm{CD}^{+}{ }^{+} \mathrm{T}$ cells into naive $\left(\mathrm{CD} 45 \mathrm{RO}-\mathrm{CCR} 7^{+} ; \mathrm{TN}\right)$ and memory $\left(\mathrm{CD}^{2} 5 \mathrm{RO}^{+}\right.$; TM) subsets, as previously described. ${ }^{55}$ In addition, a portion of the $\mathrm{CD}^{+}{ }^{+} \mathrm{T}$ cells were also activated using anti-CD3 +anti-CD28 beads (Invitrogen) and expanded in interleukin-2 (IL2) culture for 2 weeks to prepare effector T cells (TE). CD8 ${ }^{+} \mathrm{T}$ cells and $B$ cells were also purified from the same donors using Dynabead (Invitrogen) positive selection. CD4 ${ }^{+}$T-cell subsets and $B$ cells were then incubated with different concentrations of 5-ARU for $4 \mathrm{~h}$, washed and then added on to autologous PBMCs. In addition, we also added 5-ARU directly onto PBMCs or purified $\mathrm{CD}^{+}{ }^{\mathrm{T}}$ cells, which contain majority of primary MAIT cells. After 2 days, activation of primary MAIT cells within PBMC was determined through staining with MAIT cell markers and expression of CD25 and CD69 (Fig. 8a). Similar to bacterial supernatant stimulations, we found that T-cell subsets precultured with 5-ARU stimulated primary MAIT cells in a concentration-dependent manner (Fig. 8b). However, naive T cells required high concentration of 5-ARU and had the lowest capacity of activating MAIT cells, compared to memory or effector T cells (Fig. 8b). On the other hand, 5-ARU directly applied to PBMCs (that contain APCs such as dendritic cells and macrophages) or presented by purified primary B cells was relatively stronger in MAIT cell activation compared with T-cell subsets used as APCs (Fig. 8b). As expected, T2 cells had the highest stimulatory capacity (Fig. 8b).

We next determined whether stimulation of MAIT cells through T-cell presentation of metabolites would also induce effector functions and determined how this compared to B cells or direct addition to PBMCs. Accordingly, in the same experiments described above, after co-culture of 5-ARU presenting cells with PBMCs, MAIT cells were stained after $22 \mathrm{~h}$ with surface markers and for intracellular expression of tumor necrosis factor (TNF), granzyme B (GzmB) and IFNץ. As shown in representative flow cytometer data (Fig. 8c), sizeable portion of MAIT cells expressed both GzmB and TNF when 5-ARU was added directly to PBMCs. Concordant with results from the cell surface activation (Fig. 8b), memory or effector T cells induced lower levels of GzmB or TNF or IFNy compared to B-cell presentation or direct activation of PBMCs (Fig. 8c, d), and that GzmB or cytokine expression from naive $T$ cells was either very low or undetectable (Fig. 8c, d). Taken together, we conclude that primary $\mathrm{T}$ cells, particularly memory and effector T cells, have the capacity to stimulate MAIT cells in antigen-specific and MR1-restricted manner.

\section{DISCUSSION}

MAIT cells selectively respond to a broad range of microorganisms that possess the biosynthetic pathway for riboflavin metabolism. ${ }^{3,5,6,8}$ Because diverse bacterial species produce riboflavin metabolites, we hypothesized that MAIT cells can fine-tune their activation through strength of TCR signaling. The in vitro MAIT-
TCR activation assay that we developed here enabled us to test nearly four dozen bacterial species from different phyla that were primarily commensals in the human microbiota, many of which are known colonizers of mucosal tissues or skin or pathogenic species. We found highly significant variance among bacterial species for their MAIT-TCR stimulatory capacity. Furthermore, we provide evidence that T cells can act as APCs for antigen-specific activation of MAIT cells and tuning of their effector functions.

Remarkably, most bacterial species that encode the riboflavin pathway and stimulated eMAIT-TCR-expressing T cells belonged primarily to the Bacteroidetes and Proteobacteria phyla, whereas those with the lowest or no stimulatory activity were mostly from Firmicutes and a few Actinobacteria. This dichotomy is striking because the distribution or proportion of these bacterial species within the human microbiota also shifts with life history and different disease states, and these shifts may correlate with changes in MAIT cell numbers and activity. For example, the ratio of Bacteroidetes to Firmicutes species was found to be much higher in the infant gut microbiota compared to adults, and this composition skews toward an adult configuration within the first few years of life. ${ }^{56}$ Therefore, our findings on higher MAIT-TCR stimulatory capacity of Bacteroidetes relative to Firmicutes would be consistent with the notion that they may drive expansion of MAIT cells after birth. We also note that Cloacibacterium spp., species with the highest stimulatory capacity in our assay, have also been detected in breast milk samples of healthy women, ${ }^{57}$ which is one mechanism by which Bacteroidetes and other microbiota are established in the intestinal flora of infants. Moreover, the Bacteroidetes-to-Firmicutes ratio is particularly perturbed in patients with Crohn's disease, who exhibit an increased Bacteroidetes-to-Firmicutes ratio, ${ }^{26-28}$ as well as an increase in Cloacibacterium spp. in submucosal intestinal tissues. ${ }^{58}$ Importantly, biopsied inflamed tissues of Crohn's disease patients exhibit significantly higher frequency of MAIT cells than do healthy tissues, and the MAIT cells isolated from these patients secreted more pro-inflammatory IL-17 cytokine relative to the controls. ${ }^{23}$ This correlation between increased Bacteroidetes levels and MAIT cell number and activity taken together with our findings that supernatants of Bacteroidetes species displayed the highest eMAIT-TCR stimulatory activity provides a striking hypothesis: we envision that the chronic inflammation that typifies Crohn's disease might arise from perpetual activation of MAIT cells by species of the Bacteroidetes phylum, including Cloacibacterium spp. in the gut. Thus, we speculate that the bacterial species from Bacteroidetes and Proteobacteria phyla play an important role in expansion, maturation and homeostasis of MAIT cells in healthy humans and perturbation and aberrant activation of MAIT cells in inflammatory diseases.

HIV infection is another example of such a shift in microbial dysbiosis especially in the gastrointestinal track, which is also a primary site for viral replication and critical for its pathogenesis. ${ }^{59}$ Numerous reports have shown potential dysbiosis in gut microbiota during HIV infection. ${ }^{37-41}$ Interestingly, we and others have found significant decrease in MAIT cells in HIV-infected subjects, both in adults ${ }^{31-35}$ and children. ${ }^{36} \mathrm{~A}$ recent study also found that compared to healthy controls, the proportion of Firmicutes species is increased and, conversely, the proportion of Bacteroidetes is decreased in patients with HIV. ${ }^{60}$ Thus, it is tempting to speculate that the increase in ratio of low stimulators to high stimulators (Firmicutes to Bacteroidetes) of MAIT cells in HIV infection could be one of the reasons for the observed decline of MAIT cells in the blood of HIV-infected subjects.

In addition to the gut, $\mathrm{CD} 8^{+}$MAIT cells have also been shown to be resident in normal skin and are thought to play a role in skinassociated inflammations, such as psoriasis and dermatitis herpetimorfis, likely due to their determined IL-17 secretion in inflamed tissues. ${ }^{61,62}$ Indeed, similar to Crohn's disease, patients with psoriasis show an increase in the relative abundance of 
Bacteroidetes and Proteobacteria and a relative decrease in Actinobacteria and Firmicutes compared to normal skin. ${ }^{29,30}$ Of interest, Propionibacterium acnes, a member of Actinobacteria, had low/no MAIT-stimulatory capacity in our assays and is decreased in relative abundance on psoriatic skin lesions. ${ }^{29,63}$ This is especially striking since $P$. acnes is a dominant commensal member of the human skin microbiota ${ }^{64-66}$ and is thought to have a protective role in maintaining healthy skin. ${ }^{29}$ However, $P$. acnes can also cause acne when dysbiotic in oily intra-follicular skin sites. ${ }^{66-68}$ Studies of acne lesions showed that $P$. acnes induced secretion of IL-17 from infiltrated $\mathrm{CD}^{+}$or $\mathrm{CD}^{+}{ }^{+}$cells. ${ }^{69,70}$ Thus, it is conceivable that environmental signals specific to a proinflammatory environment induce the riboflavin pathway in $P$. acnes, consequently stimulating the MAIT population and causing an inflammatory reaction.

It is also plausible that the high donor-to-donor variation in MAIT cell frequencies, which can be up to 20-fold difference in healthy adults, ${ }^{1,2,71-73}$ could result from variation in the Bacteroidetes-to-Firmicutes ratio of the donor microbiota. We suggest that an individuals' unique microbiota composition and dynamics, which vary over one's lifetime ${ }^{56}$ and can change dramatically in response to different life events like pathogen response or inflammation, could be a key determinant in setting the MAIT cell frequency. For example, changes in the abundance of Bacteroidetes and Firmicutes during adulthood could be a set point for MAIT cell frequency as a sensor for microbial community composition. This is also supported by studies showing that the absence of MAIT cells in germ-free mice could be reversed upon reconstitution of the mice with bacteria. ${ }^{4,5}$ In addition to composition of the microbiota, the quantitative and qualitative differences in the magnitude of MAIT-TCR stimulation and expression of MR1 may act to fine-tune MAIT cell activation for personalized set points for their frequencies. In this regard, it was surprising that majority of $V \beta$ segments paired with canonical invariant MAIT-TCR, Va7.2 and Ja33, ${ }^{72,73}$ were non-functional. We speculate that this may partly be due to $J \beta$ segment, which we kept constant for practical purposes. However, the functional eMAIT-TCRs with V $\beta 2$ (TRBV20-1) and V $\beta 13$ (TRBV6) segments in our assay are also highly represented within primary MAIT cells, suggesting that these $V \beta$ families form higher affinity MAIT-TCR in recognition of MR1 with the antigen. ${ }^{47,71,74}$ Indeed, it has been suggested that TCR beta domain preferences may have been affected by various antigens from different microorganisms or pathogens and TCR beta recognition affinities. ${ }^{5,45,46,75-78}$ For example, in cases of strong stimulators, such as $C$. normanense, more diverse $V \beta$ recombined eMAIT-TCRs may be stimulated compared to low-stimulator bacterial species. It is also possible that different $V \beta-J \beta$ segments that are present at lower frequencies within MAIT cells provide an additional facet of modulation, with different strength of signals via more diverse and/or rarer metabolites. Functional analysis of the MAIT cells, their TCR repertoire and composition of bacterial ecosystem in large set of individuals-especially in inflammatory diseaseswould be valuable in future studies to address these possibilities.

A puzzling and striking observation from our screening assay was that many species that encode the riboflavin biosynthetic pathway in their genomes produced low levels of riboflavin and correspondingly did not exhibit eMAIT-TCR stimulatory activity. One possible explanation for this diversity of the MAIT response to the bacterial species is that low-stimulatory species may only express riboflavin pathway genes under specific microenvironmental conditions or spatial distribution in vivo that were not mimicked in our in vitro cultures. This would provide an additional important potential regulatory mechanism for bacteria MAIT activation that is based on environmental sensing pathways and the microbial milieu. For example, some Firmicutes and Actinobacteria possess putative riboflavin transporter systems that could potentially bypass the need for de novo synthesis of riboflavin, which is regulated by flavin nucleotide riboswitch if riboflavin is present in the environment. ${ }^{79,80}$ It is also worth noting that almost half of Firmicutes (130 genomes) and most Actinobacteria species ( 21 of 23 genomes) do not encode the riboflavin pathway, ${ }^{80}$ which would predict that, regardless these species have none or very low MAIT cell stimulatory capacity. Another possibility is that there may be some intraspecies diversity such that different strains can possess unique variants that alter their MAIT-stimulatory capability. For example, riboflavin biosynthetic pathway is highly conserved across Str. pneumoniae isolates, ${ }^{81}$ and genetic differences in riboflavin operon in the characterized genomes of Str. pneumoniae isolates were also identified and could affect the MAIT cell magnitude of response in vitro and in vivo. ${ }^{82}$ Future in vivo studies, for example in humanized mouse models, or assessing different in vitro culture conditions for each bacterial species, may also reveal novel conditions or molecular switches that modulate MAIT-stimulatory metabolite secretion.

While several studies have used engineered Jurkat cell lines to test bacteria-specific MAIT-TCR stimulation, the engineered conventional primary $\mathrm{T}$ cells developed here recapitulated more physiologically relevant aspects of primary MAIT cells compared to Jurkat cell line $e^{5,8,11}$ As such, compared to primary $T$ cells transduced with MAIT-TCR, Jurkat cells did not produce significant cytokines and did not have cytotoxic effector function when activated through the TCR via MR1+riboflavin metabolites. Jurkat cells also lacked the dynamic range or sensitivity in MR1dependent activation compared to primary $T$ cells. We believe our highly sensitive and high-throughput eMAIT-TCR assay using primary human $T$ cells described here may be more valuable and sensitive in screening hundreds or thousands of bacterial strains isolated from healthy individuals and patients with chronic inflammatory diseases.

Another unexpected and important finding of our study was that $T$ cells have the direct capability to present bacterial metabolites to MAIT cells in an MR1-restricted fashion, even though MR1 expression in human T-cell subsets is barely detectable in our experiments and as previously reported. ${ }^{54}$ However, the strength of MAIT activation through T-cell antigen presentation was lower compared to professional APC, such as primary B cells or MR1-expressing T2 cells. Nevertheless, the activation signals from T-cell presentation to MAIT cells were sufficient to induce low levels of pro-inflammatory cytokines (IFNY and TNFa) and cytotoxicity-associated molecule GzmB. It is also important to note that activaton of MAIT cells by other T cells, presenting riboflavin metabolites, were detected only by highstimulator bacteria or by higher concentrations of 5-ARU. This finding suggests a scenario where if the concentration of riboflavin metabolites from certain bacterial species in the mucosal tissues or site of inflammation reaches a threshold, this can recruit surrounding T cells as additional APCs. This may in turn amplify activation and effector functions of MAIT cells and propagate the inflammatory response, especially because professional APCs may be quickly killed due to MAIT cell cytotoxicity. Indeed, other mucosal cell types such as lung epithelial cells can also express $M R 1,{ }^{3}$ suggesting that MR1 expression is ubiquitous and can be utilized by MAIT cells at high riboflavin metabolite concentrations. Alternatively, it remains to be determined whether this T-MAIT interaction may also be regulatory, due to lack of costimulatory signals, similar to conventional T-cell activation. 83,84

In summary, we show that MAIT cells can discriminate between MR1-restricted, bacteria-derived metabolites through their TCR signaling thresholds and that non-professional antigen presentation such as from MR1-expressing bystander $T$ cells can regulate their activation or functions. Our findings and the development of a robust and physiologically relevant MAIT-TCR stimulation assay paves the way to further elucidate how MAIT cells, akin to other immune cells, ${ }^{85}$ decode and are tuned by the complex and variable composition of the human microbiota. This knowledge is 
1602

the first step in establishing a dynamic interactome and framework between MAIT cells and the human microbiota for the development of therapeutic approaches to maintain a healthy immunobiotic equilibrium.

\section{MATERIALS AND METHODS}

PBMC and T-cell purification

Healthy adult blood was obtained from New York Blood Center (New York, NY). PBMCs were isolated using Ficoll-paque plus (GE Healthcare). $\mathrm{CD} 4^{+} \mathrm{T}, \mathrm{CD} 8^{+} \mathrm{T}$ and $\mathrm{CD} 19^{+} \mathrm{B}$ cells were purified using Dynal CD4-positive, CD8-positive and CD19-positive Isolation Kits (Invitrogen) respectively. $\mathrm{CD}_{4}^{+}, \mathrm{CD}^{+}{ }^{+} \mathrm{T}$ and $\mathrm{CD} 19^{+} \mathrm{B}$ cells were $>99 \%$ pure as assessed by flow cytometry staining with respective antibodies. Purified $\mathrm{CD}^{+}{ }^{+} \mathrm{T}$ cells were sorted by flow cytometry (FACSAria; BD Biosciences) based on CD45RO expression into naive $\mathrm{T}$ cells (CD4 TN) (CD45RO ${ }^{-}$) and memory T cells (CD4 TM) $\left(\mathrm{CD}_{4} \mathrm{RO}^{+}\right)$, and sorted subsets were $>98 \%$ pure. In some experiments, $\mathrm{CD}_{4}^{+} \mathrm{T}$ cells were activated using anti-CD3/antiCD28-coated beads (Invitrogen) and expanded for 2 weeks (TE). Cells were cultured at $37{ }^{\circ} \mathrm{C}$ and $5 \% \mathrm{CO}_{2}$ in complete RPMI- 1640 medium (RPMI supplemented with $10 \%$ fetal bovine serum (FBS, Atlanta Biologicals)) and $1 \%$ penicillin/streptomycin (Corning Cellgro).

Cell surface and intracellular staining and flow cytometry analysis Cells were resuspended in staining buffer (PBS $+2 \%$ FBS) and incubated with fluorochrome-conjugated antibodies for $30 \mathrm{~min}$ at $4{ }^{\circ} \mathrm{C}$. Stimulation of eMAIT-TCR ${ }^{+} \mathrm{T}$ cells was determined by staining with following antibodies: mouse constant TCR $3-\mathrm{PE}$ (eBioscience), CD3-APC/Cy7, CD69-PerCP/Cy5.5 and CD25-APC. Primary MAIT cell $\left(\mathrm{CD}^{+} \mathrm{CD}^{+} \mathrm{Va} 7.2^{+} \mathrm{CD} 161^{+}\right)$stimulation was also determined by staining with antibodies CD25, CD69 and CD4BV605, CD8-A700, Va7.2-PE and CD161-BV421 (all from Biolegend). In cytotoxicity experiments, T2 cells were labeled with Cell Trace Violet (CTV) (Invitrogen) and pre-incubated with bacteria supernatant for $4 \mathrm{~h}$. After incubation, cells were washed and added to eMAIT cells. After 2 days, cells were stained with eFluor 506conjugated Fixable viability dye (eBioscience) and eMAIT surface markers and then analyzed by flow cytometry. MR1 expression on T2 cells was assessed with anti-MR1-PE and $\beta_{2}$ m expression with anti- $\beta 2 m-A P C$ (all from Biolegend). Expression of eMAIT-TCRs was determined by expression of GFP, Va7.2 and respective V $\beta$ antibody staining (kit from Beckman Coulter).

For intracellular cytokine staining, PBMCs were stimulated with the riboflavin metabolite 5-ARU (Toronto Research Chemicals, Canada) for $16 \mathrm{~h}$ and then cultured for additional $6 \mathrm{~h}$ in the presence of GolgiStop (BD Biosciences). Cells were then washed and stained for surface markers using anti-CD3Alexa Fluor 532 (eBioscience), anti-CD4-Pacific Blue, anti-CD8Brilliant Violet 570, anti-Va7.2-PE, anti-CD161-Brilliant Violet 421 and Fixable Viability Dye-eFluor 506 (Biolegend) and permeabilized using a fixation/permeabilization buffer (eBioscience). Fixed cells were then stained intracellularly with anti-GzmBAlexa Fluor 647, anti-TNFa-PE/Dazzle 594 and anti-IFNY-APC. Cy7 (Biolegend). For MR1 blocking experiments, cells were preincubated with MR1 blocking antibody or mouse IgG2a isotype control (both from Biolegend) for $2 \mathrm{~h}$ before stimulation with 5 ARU or bacteria.

Identification of MAIT cells with MR1 tetramers was described in previous study. ${ }^{11}$ APC-conjugated human MR1 tetramers which are pre-loaded with either 5-OP-RU or 6-FP (negative control) were provided by NIH Tetramer Core Facility. Engineered MAIT-TCRexpressing $\mathrm{CD}^{+} \mathrm{T}$ or Jurkat cells were stained with hMR1 tetramers for $40 \mathrm{~min}$ at room temperature followed by mTCR $\beta$ surface staining for $30 \mathrm{~min}$. All flow cytometry analyses were performed using LSR Fortessa X-20 flow cytometer (BD
Biosciences) or SP6800 (Sony) flow cytometer and analyzed using FlowJo software (Tree Star).

\section{Preparation of bacteria supernatants}

Bacterial strains were grown to stationary phase in the appropriate medium (Table S1) and centrifuged at $4000 \mathrm{rpm}$ to pellet the cells. The supernatants were then filtered through a 0.22-micron PES filter (EMD Millipore) to remove any remaining cells, and aliquoted for testing in the MAIT cell assay. Bacterial strains were obtained from the American Type Culture Collection (ATCC), DSMZ-German Collection of microorganisms and cell culture, and BEI Resources. Anaerobic bacterial species (marked as * in Table S1) were grown in the anaerobic chamber (BACTRON, Shel Lab) with the conditions including $90 \% \mathrm{~N}_{2}, 5 \% \mathrm{H}_{2}, 5 \% \mathrm{CO}_{2}$. K. pneumoniae strain was isolated from a patient by Bo Shopsin at New York University School of Medicine. Staphylococcus epidermidis Tu3298 was provided by Michael Otto at $\mathrm{NIH}$.

Preparation of human macrophages and culture with bacteria Monocytes were purified from PBMCs of blood as previously described $^{86}$ and cultured with RPMI media with $5 \%$ human serum and granulocyte-macrophage colony-stimulating factor for 1 day at 30,000 cells/well in 96-well-plate. Selected bacteria were counted, centrifuged and resuspended in the RPMI $+5 \%$ human serum media and added to macrophage cultures at varying numbers. After $4 \mathrm{~h}$ of culture, eMAIT-TCR cells were added to the same cultures. After 2 days, IFNy was measured in the supernatants by a bead assay (Qbeads, Intellicyt) according to the manufacturer's instructions and analyzed with iQue Screener plus (Intellicyt) flow cytometer.

\section{CRISPR/Cas9 gRNAs (guide RNAs) and vectors}

LentiCRISPR_V2 (Addgene plasmid \#52961) ${ }^{87}$ was a gift from Feng Zhang. To generate gene-specific 20-nucleotide target sequences with minimum off-target scores, the online CRISPR design tool was used (which was developed by the Zhang Lab). The sequences were selected to precede $5^{\prime}$-NGG protospaceradjacent motif (PAM) sequences. The 20-nucleotide sequences $5^{\prime}$-GAACCTCGCGCCTGATCACT- $3^{\prime}$ and 5'-GAGTAGCGCGAGCA CAGCTA- $3^{\prime}$ were designed to target the MR1 gene and the $B 2 M\left(\beta_{2} \mathrm{~m}\right)$ gene, respectively. The oligonucleotides were purchased from Eurofins Genomics. Next, the oligonucleotides were annealed and cloned into the single-guide RNA scaffold through Bsmbl-Bsmbl sites in the LentiCRISPR_V2 vector. The cloned constructs were then transformed into NEB Stable Competent $E$. coli (New England Biolabs). The transformants were cultured overnight before plasmid DNA was isolated using QIAprep Spin Miniprep Kit and Qiacube (Qiagen). Successful cloning with the annealed oligo was confirmed by determining $2 \mathrm{~kb}$ difference relative to the conventional LentiCRISPR_V2 vector that lacks the oligo after digesting the vectors with both Notl and BamHI restriction enzymes and running on an agarose gel. Human MR1 transcript variant 1 (NM_001531) complementary DNA (cDNA) clone was purchased from Origene. The clone was then subcloned into lentiviral vector encoding GFP marker. ${ }^{44}$

Synthetic engineering and cloning of MAIT-TCRs

Engineered TCRs were constructed as described in Fig. 2a. Peptide sequences of the gene segments were downloaded from Ensembl Genome Browser. Transcript ID for each gene segment and sequences for $\mathrm{p} 2 \mathrm{~A}$ and $\mathrm{J} \beta 2-1$ linkages are listed in Table S2. The segments were connected in the following order: Va7.2-Ja33-mTCRa-p2A-V $\beta-J \beta 2-m T C R \beta$, converted into a single open reading frame (ORF) and synthesized by Genscript. The synthesized eMAIT-TCR ORFs with each of the 21 different V $\beta$ gene segments were subcloned into a lentiviral vector encoding GFP marker. ${ }^{44}$ 
Lentiviral production and transduction

The confirmed lentiviral constructs including MR1 or eMAIT-TCRs encoding vectors and the CRISPR constructs were co-transfected with the packaging plasmids VSVG, pLP1 and pLP2 into HEK293T cells using Lipofectamine ${ }^{T M} 3000$ (Invitrogen) according to the manufacturer's protocol. Lentiviral supernatants were collected, filtered and stored as previously described. ${ }^{44}$ Cell lines or primary $T$ cells were transduced with the lentivirus at the multiplicity of infection (MOI) of $1-5$. For primary $\mathrm{T}$ cells, lentiviruses were added to purified $\mathrm{CD}^{+}$and $\mathrm{CD} 4^{+} \mathrm{T}$-cell subsets, which were stimulated using anti-CD3/anti-CD28-coated beads (Invitrogen) in a 1:2 (bead/cell) ratio. After 3 days, the cells were selected either with $0.5-1 \mu \mathrm{g} / \mathrm{ml}$ puromycin antibiotic for 3-5 days until more than $95 \%$ of non-transduced cells were dead or sorted based on marker gene.

\section{Bacteria counting}

The Bacteria Counting Kit, which enumerates bacteria by flow cytometry, was purchased from Invitrogen (MP07277). The kit includes a nucleic acid stain that penetrates both Gram-negative and Gram-positive bacteria, producing green fluorescent signal. The kit also includes a calibrated suspension of microspheres. Signals from both the stained bacteria and the beads were detected in the green fluorescence channel and were distinguished on a plot of forward scatter versus fluorescence using a flow cytometer. The density of the bacteria in the sample was determined from the ratio of bacterial signals to microsphere signals in the fluorescence-activated cell sorting (FACS) plot as the commercial protocol suggests.

\section{E. coli riboflavin gene knockout via CRISPR/Cas9}

CRISPR approach was used to achieve gene knockdown. Respective $E$. coli ribA gene sequence was extracted from the Kyoto Encyclopedia of Genes and Genomes (KEGG) ${ }^{88}$ including 100-nucleotide upstream of the start codon. Custom-made scripts were used to design spacers targeting the start sequence of the genes (non-template strand). The spacer oligos using primers (RibA_spacer_F, 5'-AAACGTTCTTCAAATCCCACCATCG and RibA_spacer_R, 5'-AAAACGATGGTGGGATTGAAGAAC) were cloned into pdCas9. ${ }^{51}$ pdCas9 was a gift from Luciano Marraffini (Addgene plasmid \# 46569). Spacer cloning into pdCas9 was carried out with 5-alpha competent $E$. coli (NEB) as described in Addgene spacer cloning manual and the manufacturer's manual. The transformed cells were plated on tryptic soy plates with chloramphenicol. Colonies were confirmed for the presence of the spacer in pCas9 plasmid using the primer pdCas9_f (5'-GTCTGAGCAAGAAATAGGCA) binding Cas9 and the respective reverse spacer pdCas9_r (5'-CGGAATGGACGATCACACTACTC) through PCR as well as a primer binding downstream of the integrated spacer pdCas9_r and the respective forward spacer. Positive colonies were grown overnight. For the eMAIT-TCR stimulation assay supernatants were obtained after centrifugation of the overnight culture for $5 \mathrm{~min}$ at $4000 \mathrm{rpm}$ followed by sterile filtration $(0.2 \mu \mathrm{m}$ Fisherbrand Syringe Filters). Cell pellets were used for RNA extraction.

\section{RNA isolation and RT-PCR}

E. coli overnight cultures were used to extract the RNA using the Qiagen RNAeasy kit. On-column DNase digestion step was carried out according to the manufacturer's manual. To improve purity of the RNA, a second round of DNase digestion was performed by adding $1 \mu \mathrm{l}$ of DNase to each $10 \mu \mathrm{g}$ of RNA eluted using the TURBO DNA-free Kit (ThermoFisher Scientific). After $30 \mathrm{~min}$, another $1 \mu \mathrm{l}$ of DNase was added to the RNA and the reaction was quenched after $30 \mathrm{~min}$ according to the manufacturer's manual. Integrity of the RNA was checked on an agarose gel.

Reverse transcription (RT) was performed according to the manufacturer's instructions using the High-Capacity cDNA
Reverse Transcription Kit (ThermoFisher Scientific). Per sample, a reaction lacking the reverse transcriptase was included to account for DNA contamination. Then, $0.4 \mu \mathrm{g}$ of RNA was used in each reaction. QRT-PCR primers (RibA-QPCR_F, 5'-CAGCTTAAACGTGTGGCAGA and RibA-QPCR_R, 5'-CGTCACCGGTCAGA(ATTC) were designed using the Real-Time qPCR Assay tool from IDT and ordered from IDT. Off-target binding was checked by aligning the primer and spacer against the $E$. coli genome. Each sample was tested in replicates using the following protocol: $5 \mu \mathrm{l} 2 \times$ PowerUp SYBR Master Mix (Applied Biosystems), $0.25 \mu \mathrm{M}$ Primer mix $(5 \mu \mathrm{M}), 2.25 \mu \mathrm{l} \mathrm{H}_{2} \mathrm{O}$ and $2.5 \mu \mathrm{l}$ of RNA. RT-PCR was carried out using the ViiA-7 Real-Time PCR System (ThermoFisher Scientific). The cysG gene was used as the control housekeeping gene (CysG-QPCR-F, 5'-GGCGAAGAGCTGGAAACA and CysG-QPCR-R, 5'-CGTGAGTGGAATACCCGAATAG). ${ }^{89}$ The Deltadelta CT-method implemented in the QuantStudio Real-Time PCR Software (v1.1) was used to analyze the RT-PCR data.

\section{Riboflavin pathway analysis}

Riboflavin biosynthesis genes in bacteria whose genome sequences have been annotated by KEGG were identified according to the annotation in KEGG pathway map00740. For unannotated bacterial genomes, riboflavin biosynthesis genes were identified in the following steps. First, all KEGG-annotated bacteria riboflavin biosynthesis genes (KEGG orthologs K01497, K01498, K02858, K00793 and K00794) were downloaded using the KEGG REST $R$ package (version 1.14.0), and grouped according to KEGG orthology. Second, for each unannotated microbial genome, genes were predicted using PRODIGAL with default parameters. ${ }^{90}$ Third, potential riboflavin biosynthesis genes were identified by aligning the KEGG-annotated riboflavin biosynthesis genes to the predicted genes using UBLAST, ${ }^{91}$ with parameters evalue $=1 e-9$ and $a c c e l=0.6$. Next, top hits from the blast search were validated using SMARTBLAST based on consistency in domain predictions. Finally, bacterial species that contain KEGG orthologs K01497, K01498, K02858, K00793 and K00794 are classified as species that have the riboflavin biosynthesis pathway.

\section{Mass spectrometry quantitation of riboflavin in bacterial} supernatants

Bacteria supernatant in PBS was acidified with $20 \%$ formic acid in water. Stable isotopic riboflavin- ${ }^{13} \mathrm{C}_{1}^{15} \mathrm{~N}_{2}$ (VB2; Toronto Research Chemicals, Canada) was spiked as the internal standard. The spiked supernatants were desalted using an Oasis HLB 96-well $\mu$ Elution plate (Waters, Waltham, MA), following the manufacturer's recommended procedures. Each sample was eluted with $50 \mu \mathrm{l}$ of the elution solution, concentrated and reconstituted to 20 $\mu \mathrm{l}$ with $0.2 \%$ formic acid in water. Riboflavin in each sample was quantified used the method of liquid chromatography-stable isotope dilution multiple reaction monitoring mass spectrometry (LC-SID-MRM MS) ${ }^{92}$ with transitions of $377.15 / 172.10$, 375.15/ 198.10 and $377.15 / 243.10$ for positive ions of riboflavin and $380.10 / 173.11,380.10 / 200.06,380.10 / 246.07$ for VB2. The column (Atlantis dC18, $5 \mu \mathrm{m}, 0.3 \mathrm{~mm} \times 150 \mathrm{~mm}$; Waters, Waltham, MA) temperature was $40^{\circ} \mathrm{C}$. Solvent $A$ was water/acetonitrile/formic acid 98.8:1.0:0.2 $(\mathrm{v} / \mathrm{v} / \mathrm{v})$, solvent $B$ was water/acetonitrile/formic acid 1.0:98.8:0.2 (v/v/v) and the flow rate was $9 \mu \mathrm{l} / \mathrm{min}$. Each sample was analyzed in triplicate. Chromatograms were processed using the Skyline software and ratios of the peak area for riboflavin to that for VB2 were obtained. Original concentrations of riboflavin in bacterial supernatants were calculated using these ratios and the absolution concentration of VB2 in the analysis samples. Limit of quantitation for riboflavin was determined using the method of "blank and low-concentration samples". ${ }^{93}$ PBS treated with the same desalting procedure was used as the blank $^{94}$. 


\section{Statistical analysis}

Correlations with Spearman's rank test and two-tailed Mann-Whitney U-tests were performed using GraphPad Prism software. No outliers were excluded in any of the statistical tests. Scatter dot plots report the median center lines with 25th and 75th quartile (interquartile range), and each data point represents an independent measurement. Correlation analysis was assessed with Spearman's rank test. Bar plots report the mean and standard deviation or the standard error of the mean. Threshold of significance for all tests was set at $<0.05$.

\section{ACKNOWLEDGEMENTS}

We thank Dr. David Mellert (Jackson Laboratory) for critical reading and critiques, Dr. Victor Torres (NYU School of Medicine) and Dr. Bo Shopsin (NYU School of Medicine) for insightful discussions and suggestions, Drs. Victor Torres, Bo Shopsin, Michael Otto (NIH) and George Weinstock (Jackson Laboratory) for several bacteria strains, and NIH tetramer facility for the MR1 tetramers. The research in this study was supported by the National Institute of Health (NIH) grant R01Al121920 to D.U, NIH Grant U54NS105539 to D.U., J.O. and X.Y. and the Jackson Laboratory Director's Innovation Fund to D.U and J.O.

\section{AUTHOR CONTRIBUTIONS}

C.T. designed, performed and analyzed the experiments. W.Z. and J.O. performed bioinformatics studies. A.Y.V. performed CRISPR-repression experiments in E. coli. E.K. helped T-T interaction assays and performed intracellular staining. M.H., L.P., E.K. and L.K. prepared human primary $T$ cells for the experiments. E.F. performed growth of the bacterial species. M.H. and L.K. isolated and prepared healthy human adult PBMCs. X.Y and L.W. performed mass spec analysis of riboflavin in bacteria supernatants. W.Z., A.Y.V, M.H., E.F., L.P., E.K. and L.K. provided helpful discussions in experimental design. C.T., J.O. and D.U. wrote the manuscript. J.O. and D.U. led the investigation and contributed to the design and interpretation of the data.

\section{ADDITIONAL INFORMATION}

The online version of this article (https://doi.org/10.1038/s41385-018-0072-x) contains supplementary material, which is available to authorized users.

Competing interests: The authors declare no competing interests.

\section{REFERENCES}

1. Dusseaux, M. et al. Human MAIT cells are xenobiotic-resistant, tissue-targeted, CD161hi IL-17-secreting T cells. Blood 117, 1250-1259 (2011).

2. Martin, E. et al. Stepwise development of MAIT cells in mouse and human. PLoS Biol. 7, e54 (2009).

3. Gold, M. C. et al. Human mucosal associated invariant T cells detect bacterially infected cells. PLoS Biol. 8, e1000407 (2010).

4. Treiner, E. et al. Selection of evolutionarily conserved mucosal-associated invariant T cells by MR1. Nature 422, 164-169 (2003).

5. Le Bourhis, L. et al. Antimicrobial activity of mucosal-associated invariant T cells. Nat. Immunol. 11, 701-708 (2010).

6. Meierovics, A., Yankelevich, W. J. \& Cowley, S. C. MAIT cells are critical for optimal mucosal immune responses during in vivo pulmonary bacterial infection. Proc. Natl Acad. Sci. USA 110, E3119-3128 (2013).

7. Chua, W. J. et al. Polyclonal mucosa-associated invariant $T$ cells have unique innate functions in bacterial infection. Infect. Immun. 80, 3256-3267 (2012).

8. Kjer-Nielsen, L. et al. MR1 presents microbial vitamin B metabolites to MAIT cells. Nature 491, 717-723 (2012).

9. Patel, O. et al. Recognition of vitamin B metabolites by mucosal-associated invariant T cells. Nat. Commun. 4, 2142 (2013).

10. Lopez-Sagaseta, J. et al. The molecular basis for Mucosal-Associated Invariant T cell recognition of MR1 proteins. Proc. Natl Acad. Sci. USA 110, E1771-1778 (2013).

11. Corbett, A. J. et al. T-cell activation by transitory neo-antigens derived from distinct microbial pathways. Nature 509, 361-365 (2014).

12. Le Bourhis, L. et al. MAIT cells detect and efficiently lyse bacterially-infected epithelial cells. PLoS Pathog. 9, e1003681 (2013).

13. Kurioka, A. et al. MAIT cells are licensed through granzyme exchange to kill bacterially sensitized targets. Mucosal Immunol. 8, 429-440 (2015).

14. Cui, Y. et al. Mucosal-associated invariant T cell-rich congenic mouse strain allows functional evaluation. J. Clin. Invest. 125, 4171-4185 (2015).
15. Georgel, P., Radosavljevic, M., Macquin, C. \& Bahram, S. The non-conventional MHC class I MR1 molecule controls infection by Klebsiella pneumoniae in mice. Mol. Immunol. 48, 769-775 (2011).

16. Ussher, J. E., Klenerman, P. \& Willberg, C. B. Mucosal-associated invariant T-cells: new players in anti-bacterial immunity. Front. Immunol. 5, 450 (2014).

17. Liuzzi, A. R. et al. Unconventional human T cells accumulate at the site of infection in response to microbial ligands and induce local tissue remodeling. $J$. Immunol. 197, 2195-2207 (2016).

18. Jiang, J. et al. Mucosal-associated invariant T-cell function is modulated by programmed death-1 signaling in patients with active tuberculosis. Am. J. Respir. Crit Care Med. 190, 329-339 (2014).

19. Grimaldi, D. et al. Specific MAIT cell behaviour among innate-like T lymphocytes in critically ill patients with severe infections. Intensive Care Med. 40, 192-201 (2014).

20. Smith, D. J., Hill, G. R., Bell, S. C. \& Reid, D. W. Reduced mucosal associated invariant T-cells are associated with increased disease severity and Pseudomonas aeruginosa infection in cystic fibrosis. PLOS ONE 9, e109891 (2014).

21. Leung, D. T. et al. Circulating mucosal associated invariant T cells are activated in Vibrio cholerae $\mathrm{O} 1$ infection and associated with lipopolysaccharide antibody responses. PLoS Negl. Trop. Dis. 8, e3076 (2014).

22. Booth, J. S. et al. Mucosal-associated invariant T cells in the human gastric mucosa and blood: role in Helicobacter pylori infection. Front. Immunol. 6, 466 (2015).

23. Serriari, N. E. et al. Innate mucosal-associated invariant T (MAIT) cells are activated in inflammatory bowel diseases. Clin. Exp. Immunol. 176, 266-274 (2014).

24. Illes, Z., Shimamura, M., Newcombe, J., Oka, N. \& Yamamura, T. Accumulation of Valpha7.2-Jalpha33 invariant $T$ cells in human autoimmune inflammatory lesions in the nervous system. Int. Immunol. 16, 223-230 (2004).

25. Cho, Y. N. et al. Mucosal-associated invariant T cell deficiency in systemic lupus erythematosus. J. Immunol. 193, 3891-3901 (2014).

26. Wright, E. K. et al. Recent advances in characterizing the gastrointestinal microbiome in Crohn's disease: a systematic review. Inflamm. Bowel Dis. 21, 1219-1228 (2015).

27. Walker, A. W. et al. High-throughput clone library analysis of the mucosaassociated microbiota reveals dysbiosis and differences between inflamed and non-inflamed regions of the intestine in inflammatory bowel disease. BMC Microbiol. 11, 7 (2011).

28. Andoh, A. et al. Comparison of the fecal microbiota profiles between ulcerative colitis and Crohn's disease using terminal restriction fragment length polymorphism analysis. J. Gastroenterol. 46, 479-486 (2011).

29. Drago, L. et al. Skin microbiota of first cousins affected by psoriasis and atopic dermatitis. Clin. Mol. Allergy 14, 2 (2016).

30. Fahlen, A., Engstrand, L., Baker, B. S., Powles, A. \& Fry, L. Comparison of bacterial microbiota in skin biopsies from normal and psoriatic skin. Arch. Dermatol. Res. 304, 15-22 (2012).

31. Cosgrove, C. et al. Early and nonreversible decrease of CD161++/MAIT cells in HIV infection. Blood 121, 951-961 (2013).

32. Wong, E. B. et al. Low levels of peripheral CD161++CD8+ mucosal associated invariant T (MAIT) cells are found in HIV and HIV/TB co-infection. PLOS ONE 8, e83474 (2013).

33. Eberhard, J. M. et al. CD161+ MAIT cells are severely reduced in peripheral blood and lymph nodes of HIV-infected individuals independently of disease progression. PLOS ONE 9, e111323 (2014).

34. Fernandez, C. S. et al. MAIT cells are depleted early but retain functional cytokine expression in HIV infection. Immunol. Cell Biol. 93, 177-188 (2015).

35. Leeansyah, E. et al. Activation, exhaustion, and persistent decline of the antimicrobial MR1-restricted MAIT-cell population in chronic HIV-1 infection. Blood 121, 1124-1135 (2013).

36. Khaitan, A. et al. HIV-infected children have lower frequencies of CD8+ mucosalassociated invariant T (MAIT) cells that correlate with innate, Th17 and Th22 cell subsets. PLOS ONE 11, e0161786 (2016).

37. Gori, A. et al. Early impairment of gut function and gut flora supporting a role for alteration of gastrointestinal mucosa in human immunodeficiency virus pathogenesis. J. Clin. Microbiol. 46, 757-758 (2008).

38. Round, J. L. \& Mazmanian, S. K. The gut microbiota shapes intestinal immune responses during health and disease. Nat. Rev. Immunol. 9, 313-323 (2009).

39. Vujkovic-Cvijin, I. et al. Dysbiosis of the gut microbiota is associated with HIV disease progression and tryptophan catabolism. Sci. Transl. Med. 5, 193 ra191 (2013).

40. Lozupone, C. A. et al. Alterations in the gut microbiota associated with HIV-1 infection. Cell Host Microbe 14, 329-339 (2013).

41. Nowak, P. et al. Gut microbiota diversity predicts immune status in HIV-1 infection. AIDS 29, 2409-2418 (2015).

42. Henderson, R. A. et al. HLA-A2.1-associated peptides from a mutant cell line: a second pathway of antigen presentation. Science 255, 1264-1266 (1992). 
Tuning of human MAIT cell activation by commensal bacteria species and... C Tastan et al.

43. Cohen, C. J., Zhao, Y., Zheng, Z., Rosenberg, S. A. \& Morgan, R. A. Enhanced antitumor activity of murine-human hybrid T-cell receptor (TCR) in human lymphocytes is associated with improved pairing and TCR/CD3 stability. Cancer Res. 66, 8878-8886 (2006).

44. Wan, Q. et al. Probing the effector and suppressive functions of human $T$ cell subsets using antigen-specific engineered T cell receptors. PLOS ONE 8, e56302 (2013).

45. Gold, M. C. et al. MR1-restricted MAIT cells display ligand discrimination and pathogen selectivity through distinct $\mathrm{T}$ cell receptor usage. J. Exp. Med. 211, 1601-1610 (2014).

46. Gherardin, N. A. et al. Diversity of T cells restricted by the MHC class I-related molecule MR1 facilitates differential antigen recognition. Immunity 44, 32-45 (2016).

47. Lepore, M. et al. Parallel T-cell cloning and deep sequencing of human MAIT cells reveal stable oligoclonal TCRbeta repertoire. Nat. Commun. 5, 3866 (2014).

48. Aldemir, H. Novel MHC class I-related molecule MR1 affects MHC class I expression in 293T cells. Biochem. Biophys. Res. Commun. 366, 328-334 (2008).

49. Yamaguchi, H. \& Hashimoto, K. Association of MR1 protein, an MHC class I-related molecule, with beta(2)-microglobulin. Biochem. Biophys. Res. Commun. 290 722-729 (2002)

50. Soudais, C. et al. In vitro and in vivo analysis of the Gram-negative bacteriaderived riboflavin precursor derivatives activating mouse MAIT cells. J. Immunol. 194, 4641-4649 (2015).

51. Bikard, D. et al. Programmable repression and activation of bacterial gene expression using an engineered CRISPR-Cas system. Nucleic Acids Res. 41, 7429-7437 (2013).

52. Oh, J. et al. The altered landscape of the human skin microbiome in patients with primary immunodeficiencies. Genome Res. 23, 2103-2114 (2013).

53. Human Microbiome Project Consortium. Structure, function and diversity of the healthy human microbiome. Nature 486, 207-214 (2012).

54. McWilliam, H. E. et al. The intracellular pathway for the presentation of vitamin Brelated antigens by the antigen-presenting molecule MR1. Nat. Immunol. 17, 531-537 (2016).

55. Sundrud, M. S. et al. Genetic reprogramming of primary human T cells reveals functional plasticity in Th cell differentiation. J. Immunol. 171, 3542-3549 (2003).

56. Mariat, D. et al. The Firmicutes/Bacteroidetes ratio of the human microbiota changes with age. BMC Microbiol. 9, 123 (2009).

57. Urbaniak, C. et al. Effect of chemotherapy on the microbiota and metabolome of human milk, a case report. Microbiome 2, 24 (2014).

58. Chiodini, R. J. et al. Microbial population differentials between mucosal and submucosal intestinal tissues in advanced Crohn's disease of the ileum. PLOS ONE 10, e0134382 (2015).

59. Mehandru, S., Tenner-Racz, K., Racz, P. \& Markowitz, M. The gastrointestinal tract is critical to the pathogenesis of acute HIV-1 infection. J. Allergy Clin. Immunol. 116, 419-422 (2005).

60. Ling, Z. et al. Alterations in the fecal microbiota of patients with HIV-1 infection: an observational study in a Chinese population. Sci. Rep. 6, 30673 (2016).

61. Teunissen, M. B. et al. The IL-17A-producing CD8+ T-cell population in psoriatic lesional skin comprises mucosa-associated invariant $T$ cells and conventional T cells. J. Invest. Dermatol. 134, 2898-2907 (2014).

62. Li, J. et al. The frequency of mucosal-associated invariant T cells is selectively increased in dermatitis herpetiformis. Australas. J. Dermatol. 58, 200-204 (2017).

63. Gao, Z., Tseng, C. H., Strober, B. E., Pei, Z. \& Blaser, M. J. Substantial alterations of the cutaneous bacterial biota in psoriatic lesions. PLOS ONE 3, e2719 (2008).

64. Leeming, J. P., Holland, K. T. \& Cunliffe, W. J. The microbial ecology of pilosebaceous units isolated from human skin. J. Gen. Microbiol. 130, 803-807 (1984).

65. Kong, H. H. et al. Temporal shifts in the skin microbiome associated with disease flares and treatment in children with atopic dermatitis. Genome Res. 22, 850-859 (2012).

66. Grice, E. A. et al. Topographical and temporal diversity of the human skin microbiome. Science 324, 1190-1192 (2009).

67. Beylot, C. et al. Propionibacterium acnes: an update on its role in the pathogenesis of acne. J. Eur. Acad. Dermatol. Venereol. 28, 271-278 (2014).

68. Leyden, J. J., McGinley, K. J. \& Vowels, B. Propionibacterium acnes colonization in acne and nonacne. Dermatology 196, 55-58 (1998).

69. Kistowska, M. et al. Propionibacterium acnes promotes Th17 and Th17/Th1 responses in acne patients. J. Invest. Dermatol. 135, 110-118 (2015).
70. Kelhala, H. L. et al. IL-17/Th17 pathway is activated in acne lesions. PLoS ONE 9, e105238 (2014)

71. Held, K., Beltran, E., Moser, M., Hohlfeld, R. \& Dornmair, K. T-cell receptor repertoire of human peripheral CD161hiTRAV1-2+ MAIT cells revealed by next generation sequencing and single cell analysis. Hum. Immunol. 76, 607-614 (2015).

72. Tilloy, F. et al. An invariant $T$ cell receptor alpha chain defines a novel TAPindependent major histocompatibility complex class lb-restricted alpha/beta $T$ cell subpopulation in mammals. J. Exp. Med. 189, 1907-1921 (1999).

73. Reantragoon, R. et al. Antigen-loaded MR1 tetramers define T cell receptor heterogeneity in mucosal-associated invariant T cells. J. Exp. Med. 210, 2305-2320 (2013).

74. Kitaura, K., Shini, T., Matsutani, T. \& Suzuki, R. A new high-throughput sequencing method for determining diversity and similarity of T cell receptor (TCR) alpha and beta repertoires and identifying potential new invariant TCR alpha chains. BMC Immunol. 17, 38 (2016).

75. Eckle, S. B. et al. A molecular basis underpinning the T cell receptor heterogeneity of mucosal-associated invariant T cells. J. Exp. Med. 211, 1585-1600 (2014).

76. Howson, L. J. et al. MAIT cell clonal expansion and TCR repertoire shaping in human volunteers challenged with Salmonella Paratyphi A. Nat. Commun. 9, 253 (2018).

77. Dias, J., Leeansyah, E. \& Sandberg, J. K. Multiple layers of heterogeneity and subset diversity in human MAIT cell responses to distinct microorganisms and to innate cytokines. Proc. Natl Acad. Sci. USA 114, E5434-E5443 (2017)

78. Lopez-Sagaseta, J. et al. MAIT recognition of a stimulatory bacterial antigen bound to MR1. J. Immunol. 191, 5268-5277 (2013).

79. Gutierrez-Preciado, A. et al. Extensive identification of bacterial riboflavin transporters and their distribution across bacterial species. PLOS ONE 10, e0126124 (2015).

80. Magnusdottir, S., Ravcheev, D., de Crecy-Lagard, V. \& Thiele, I. Systematic genome assessment of B-vitamin biosynthesis suggests co-operation among gut microbes. Front. Genet. 6, 148 (2015).

81. Kurioka, A. et al. Diverse Streptococcus pneumoniae strains drive a mucosalassociated invariant T-cell response through major histocompatibility complex class I-related molecule-dependent and cytokine-driven pathways. J. Infect. Dis. 217, 988-999 (2018)

82. Hartmann, N. et al. Riboflavin metabolism variation among clinical isolates of Streptococcus pneumoniae results in differential activation of mucosalassociated invariant T cells. Am. J. Respir. Cell Mol. Biol. 58, 767-776 (2018).

83. Zheng, Y., Zha, Y. \& Gajewski, T. F. Molecular regulation of T-cell anergy. EMBO Rep. 9, 50-55 (2008).

84. Smith, T. R., Verdeil, G., Marquardt, K. \& Sherman, L. A. Contribution of TCR signaling strength to CD8+ T cell peripheral tolerance mechanisms. J. Immunol. 193, 3409-3416 (2014).

85. Pamer, E. G. Microbial tuning of the mammalian immune system. Trends Mol. Med. 23, 379-380 (2017).

86. Manel, N. et al. A cryptic sensor for HIV-1 activates antiviral innate immunity in dendritic cells. Nature 467, 214-217 (2010).

87. Sanjana, N. E., Shalem, O. \& Zhang, F. Improved vectors and genome-wide libraries for CRISPR screening. Nat. Methods 11, 783-784 (2014).

88. Kanehisa, M. et al. KEGG for linking genomes to life and the environment. Nucleic Acids Res. 36(Database issue), D480-484 (2008).

89. Zhou, K. et al. Novel reference genes for quantifying transcriptional responses of Escherichia coli to protein overexpression by quantitative PCR. BMC Mol. Biol. 12, 18 (2011).

90. Hyatt, D. et al. Prodigal: prokaryotic gene recognition and translation initiation site identification. BMC Bioinforma. 11, 119 (2010).

91. Edgar, R. C. Search and clustering orders of magnitude faster than BLAST. Bioinformatics 26, 2460-2461 (2010).

92. Ciccimaro, E. \& Blair, I. A. Stable-isotope dilution LC-MS for quantitative biomarker analysis. Bioanalysis 2, 311-341 (2010).

93. Mani, D. R., Abbatiello, S. E. \& Carr, S. A. Statistical characterization of multiplereaction monitoring mass spectrometry (MRM-MS) assays for quantitative proteomics. BMC Bioinforma. 13(Suppl. 16), S9 (2012).

94. Reantragoon, R. et al. Structural insight into MR1-mediated recognition of the mucosal associated invariant $T$ cell receptor. J. Exp. Med. 209, 761-774 (2012). 